\title{
Optimal auctions with externalities and signaling*
}

\author{
Jozsef Molnar ${ }^{\dagger}$ and Gabor Virag ${ }^{\ddagger}$
}

June 18, 2001

\begin{abstract}
This paper asks how to design a revenue-maximizing auction in the presence of post-auction market competition and asymmetric information. In this situation, bidders, depending on the auction mechanism, can signal their type through their bidding behavior which influences the outcome of the post-auction market game. Thus the auctioneer has to design a mechanism that not only specifies who wins the good but also describes the amount of information that can be revealed about the winner through signaling. This paper shows that to reveal all information about the winner can be optimal under some conditions.
\end{abstract}

\footnotetext{
${ }^{*}$ We thank to Jacques Cremer, Jeffrey Eli, Peter Eso, Phillipe Jehiel, Robert Porter, William Rogerson, Yossi Spiegel, Asher Wolinsky, Michael Whinston for their helpful comments. All remaining errors are ours.

${ }^{\dagger}$ Affiliation: Department of Economics, Northwestern University. 2003 Sheridan Road, Evanston IL 60208. E-mail: j-molnar@nwu.edu

${ }^{\ddagger}$ Department of Economics, Princeton University, Fisher Hall 001, Princeton, NJ 08544 E-mail: gvirag@princeton.edu
} 


\section{Introduction}

It is usually assumed in the auction literature that the only relevant private information of the bidders concerns their valuation and that they do not care whether this information is revealed or not to outside parties (or other bidders) through the auction process. In several cases of real world auctions this assumption does not hold. Examples include takeover battles, bidding for patented innovation that can reduce production cost or auctioning licenses to operate in new markets (e.g., the $3 \mathrm{G}$ license auctions).

In this paper we consider two examples. In the first example $n$ bidders compete for a licence to enter to a market where the price is regulated. There are informed and uninformed consumers on the market. In addition to the decision about who wins the license the government has also to decide what signal he should send to the uninformed consumers about the quality level of the product of the winner (which he discovers in the auction process). We assume that the government (the seller) can commit to an information transmission mechanism perfectly and that the uninformed customers are unable to observe the bids of the competitors. Then the main question is how he can maximize his revenue, in particular should he always announce the type of the winner or should he hide some information.

Our second example is a takeover battle. Consider three quantity-setting firms that produce a homogenous product. One of the three firms can be taken over by one of the other two firms in an auction. After the auction the remaining two firms continue to compete in the product market in quantities but the cost of the merged firm remains private information. The premium paid for the target firm could also convey information about the winner firm to the rival, which affects the rival's post-auction market behavior. Conditional on losing the auction, the payoff of the losing bidder will not be zero. The willingness to pay of any given bidder will depend on the identity of the winner and the informativeness of the mechanism. In this case the profit function of the bidders does not satisfy our earlier additive separability assumption. We show that, in this particular example, in the optimal mechanism all information will be revealed about the winning bidder.

Besides analyzing how the possibilities of information transmission to third parties alter the bidding behavior, our main aim in this paper is to make a modest step in 
characterizing the optimal mechanism in this new, somewhat broader environment.

In the mechanism design literature Pavan and Calzolari (2001) consider a similar model to ours. They examine a contracting environment where two principals sequentially interact with a common agent. They endogenize the information flow between the two contractual relationship and show how an upstream principal may benefit from designing a mechanism that strategically discloses information to a downstream principal: By providing the receiver with a signal that is correlated with the agent's type and his past contractual experiment, the upstream principal succeeds in appropriating part of the agent's surplus from downstream contracting. Our model considers a simultaneous decision making game in the second stage and continuous type space. Lizzeri (1999) considers a model of certification institutes that after (a costless) investigation can certify the product quality of a firm that applied. The basic question in that paper concerns the features of the disclosure policy that is revenue maximizing for the institute. He shows that no disclosure of any information may well be profit maximizing for the institute. In our model we do not allow costless observation of the type of the applicants so we need to rely on direct revelation mechanisms to extract this information. Another difference is that we consider convex/concave functional forms while Lizzeri (1999) only considers the linear case. We are able to show in this paper that if we use a revelation mechanism in the Lizzeri framework then every disclosure policy maximize profits.

Our paper is also related to the literature of auctions with externalities initiated by Jehiel and Moldovanu. See for example Jehiel-Moldovanu (1997), (1998) and (2000) ${ }^{1}$. The main idea is to allow for the possibility that other market participants (bidders or just outside players) are influenced by who wins the object and at what price. The externality can be allocative, i.e. the utility of the other bidders are influenced by who wins the object or it can be an informational externality; i.e. the bidders send signals that will change the beliefs of the other agents. In our example 1 if the winner is thought to produce higher quality product, then the uninformed consumers will have higher demand for the product and the winner of the license earns more profit in the post-auction market.

The seminal paper of Jehiel-Moldovanu (1997) makes the assumption that the private information of the bidders is automatically revealed after the auction process, an

\footnotetext{
${ }^{1}$ In this framework, Molnar (2000) provides a rational explanation of some horizontal mergers in which the stock value of the acquirer firm is decreasing.
} 
assumption that excludes the possibility of signaling. That assumption is not realistic but helps to avoid the difficulty of equilibrium selection in signaling games. We can not avoid this problem the same way though, because information transmission is our main concern in this paper. Since our paper has continuous types, we cannot apply commonly used refinements to eliminate unreasonable equilibria. In a similar setting Goeree (2000) allows for the possibility of signaling and "solves" the problem of multiple equilibria in first- and second-price auctions by implicitly ruling out all of the non-separating equilibria, an ad-hoc assumption. In this paper we assume that the seller is able to commit to a mechanism throughout the auction process and this mechanism is common knowledge between the bidders and the uninformed consumers (e.g. through a public announcement), and the signals will be sent to the uninformed consumers in a way that is specified by the mechanism. This both pins down the beliefs on the equilibrium path and makes it unnecessary to deal with out-of-equilibrium beliefs.

In Goeree (2000) the losing bidder is worse off if the winner is able to signal higher type through his bid. Our second example extends Goeree's analysis and shows that full information disclosure is indeed optimal in this case. ${ }^{2}$ The advantage of this approach over Goeree's is not only that it helps avoid his ad-hoc assumption about the structure of equilibria. The mechanism design approach confronts the question of how to design an optimal (revenue-maximizing) selling mechanism in the presence of signaling possibilities. Goeree analyzed only some simple mechanisms such as the first- and second-price auctions. However, because of the strong commitment power of the seller, the results of our paper must be considered as a limiting case. No matter what the seller does, he will not be able to do better than under these assumptions.

Our first example will be introduced in the next section. Section 2 explains some results of Goeree (2000) about the first- and second-price auctions and adds some results of ours. We analyze a signaling game form in this section, which makes it different from the latter parts. Section 3 introduces the mechanism design approach and characterizes optimal mechanisms. Section 4 describes our second example and Section 5 characterizes

\footnotetext{
${ }^{2}$ Molnar (2000, p.6) asks similar questions as Goeree (2000) and he introduces the concept that we will call modified second-price auction. See also the paper of Das Varma (2000) that deals with the existence of separating equilibrium of the first-price auction if we allow signalling. His emphasis is on the efficiency of the outcome while Goeree (2000) and this paper focus on revenue maximization in a wider class of auctions
} 
the optimal mechanism in this case. Section 6 concludes and discusses the main constraints of our approach and some possible generalizations for future research. Most of the proofs will be given in the Appendix.

\section{Example 1}

\subsection{The model}

There are $n$ firms competing in an auction for a single indivisible license to enter into a market where the price is regulated by the government. The winning bidder gets the license, can enter the market and sell the product to consumers who are either informed or uninformed about the quality of the product. The product's quality depend on the type of the firm and it can not be observed by the uninformed consumers. The firms are symmetric ex-ante. The distribution of their type is independent and identical, with a support $[\underline{t}, \bar{t}]$, a strictly positive density function $f$, and a distribution function $F$. We will denote bidders by $1 \ldots . n$. Let $t_{i}$ be the type of bidder $i, t=\left(t_{1}, t_{n}\right)$ be the vector of types.

The utility function of the consumers is $\theta t_{i}-P$. Where $\theta$ is the type of the consumers, distributed on $[0,1]$ with a distribution function $G$. The demand function of the consumers can be derived from their utility function. Demand is normalized to 1 where the share of the informed consumers is $\alpha$ and the share of the uninformed is $1-\alpha$.

The demand of the informed consumers is:

$$
q_{I}=\left\{\begin{array}{c}
\alpha \text { if } \theta \geq \frac{P}{t_{i}} \\
0 \text { otherwise }
\end{array}\right\}
$$

The demand of the uninformed consumers is:

$$
q_{U}=\left\{\begin{array}{c}
(1-\alpha) \text { if } \theta \geq \frac{P}{h_{i}} \\
0 \text { otherwise }
\end{array}\right\}
$$

where $h_{i}$ is the expected type of the product by the uninformed consumers.

The uninformed consumers will form their expectations based on the information what they get from the mechanism designer. The mechanism will consist of the probability of 
winning as a function of types $p_{i}(\widetilde{t})$, and expected payment $b_{i}\left(\widetilde{t}_{i}\right)$ and an information disclosure rule.

The uninformed consumers will get a message $z$ from the mechanism designer. From this message they infer the quality of the product using their updating function $h(z)$. The message may be random from the ex-ante point of view of bidder $i$ 's type $t_{i}$, but can depend, even deterministically, on the bids (or in a general mechanism the announced type) of the other bidders. We will use the term $E_{z \mid t}$ to denote the expectation of $z$ conditional on $t$.

The consumers care only about the expected value of the winners' type because of the linearity of their utility function in $t$, so $h(z)$ can be thought of as the expected value of the types (conditional on winning) that have the same signal as $t_{i}$ does. Let $S(z)$ be the set of types of some bidders that produce signal $z$ for the consumers with positive probability. Then

$$
h(z)=E_{x \in S(z)}[x \mid x \text { is winner, } z(x)=z] .
$$

To illuminate the nature of $h(z)$, consider three possible cases here. First suppose that there is full signaling in the sense that in equilibrium the type of the winner will be known surely to the seller and to the uninformed consumers. Then $h(z)=t_{i}$ surely. On the other hand, if we assume that no information will be transmitted by the auction process (or equivalently the information is hidden from the uninformed consumers) then $h(z)=h=E_{t}\left(t_{i} \mid t_{i}\right.$ is winner) for every $i$ and $t_{i}$.

Consider now an example for a random outcome. An ex-ante random $h(z)$ is given by a second-price auction mechanism where only the second highest bid is announced. Denote this second highest bid by $z$. Then $h(z)$ is the expected value of the highest type conditional on $z$. This last example shows a random mechanism that is not random in the sense that the belief function is a deterministic function of the bids. But it is random from the ex-ante point of view of type $t_{i}$ because conditional on winning he does not know what the second highest bid will be.

The profit function of the winning bidder in the second stage is the following:

$$
V\left(t_{i}, h(z)\right)=P \alpha\left(1-G\left(\frac{P}{t_{i}}\right)\right)+P(1-\alpha)\left(1-G\left(\frac{P}{h(z)}\right)\right)
$$


Assume identical quasi-linear utility function for the bidders. The expected payment of type $\widetilde{t}_{i}$ is denoted by $b_{i}\left(\widetilde{t_{i}}\right)$ and $p_{i}(\widetilde{t})$ is the probability of winning for type $\widetilde{t}_{i}$ if the others announce $\widetilde{t}_{-i}$. Then the utility of type $t_{i}$, if he reports $\widetilde{t}_{i}$ and the other types report $\widetilde{t}_{-i}$ is as follows:

$$
U\left(t_{i}, \widetilde{t}_{i}, z\right)=E_{z \mid \tilde{t}}\left[p_{i}(\widetilde{t}) V\left(t_{i}, h(z)\right)-b_{i}\left(\widetilde{t_{i}}\right)\right],
$$

The consumers play a passive role; only the uninformed consumers' beliefs will be important which are pinned down by the mechanism except in the next section where restrictive assumptions are employed to make beliefs unambiguous. So we will not need to consider the consumer's decision problem in our game.

The timing of our game is the following.

Stage 0 . The seller announces the probability of winning, the payments and the information disclosure policy. Nature choose the types of the bidders.

Stage 1. The bidders bid for the license to produce the product at a given price $P$.

Stage 2. The winning bidder produces a product of quality $t_{i}$. The uninformed consumers learn $z$ and decide whether to buy or not at price $P$.

In the next subsection we will show that different mechanisms will result in different information disclosures and different revenues for the seller. We generalize our model and show that if the second stage profit function is additively separable in the true type and the expected type our analysis go through. After that we derive the optimal mechanism in this setup.

\subsection{First- and second-price auctions with separating equilibria}

In this section we follow Goeree (2000) closely in the treatment of this topic. Because bidders are ex-ante identical it is natural to focus attention on symmetric equilibria in pure strategies. This assumption implies that in this section the strategy of the bidders is characterized by a deterministic and symmetric bid function. Hence we will not write the subscripts for players. So, here $t$ will mean a type and not the vector of all of the types. First we make some assumptions ${ }^{3}$ :

Assumption 1 (A1): $V(t, h)$ is strictly increasing in both $t$ and $h$ and thrice differentiable including all partials.

\footnotetext{
${ }^{3}$ These assumptions are satisfied in our example above.
} 
It is only a conventional ordering that $V$ is strictly increasing in the first component. However, Goeree (2000) also analyzes the case where a higher second component $(h)$ is worse. Our assumption excludes this possibility.

To reach existence of a separating equilibrium Goeree makes the following two assumptions:

Assumption 2 (A2): $\frac{F(t)}{f(t)}$ is (weakly) increasing for every $t$.

Assumption 3 (A3): The partial derivative of $V$ with respect to the second component is increasing in both components: $\left.\frac{\partial V(t, h)}{\partial h}\right|_{h=k}$ is increasing both in $t$ and in $k$.

The bidders know the auction mechanism and the disclosure rule of the seller to the consumers and they will take it into account in their bid. Define an outcome separating if every type of bidder bids differently and thus all of the incentive-compatibility constraints apply. The above assumptions (A1, A2 and A3) guarantee (see Goeree (2000)) that both the first-price and the simple second-price auction have a separating equilibrium. We use the concept of the simple second-price auction as the sealed-bid form in which all of the bids are revealed to the uninformed consumers. Because the winning bid is revealed to the consumers in both the first-price and the (simple) second-price auction the consumers will know the type of the winner surely, so $h(t)=t$ holds with probability 1. Call a separating equilibrium in which $h(t)=t$ holds with probability 1 a full-signaling separating equilibrium. So in our new terminology the separating equilibrium of both the first-price auction and the second-price auction is unique if exists and is a full-signaling separating equilibrium. We will thus refer to the separating equilibrium of these auctions both as a full-signaling separating equilibrium and simply as a separating equilibrium.

We introduce the concept of a modified second-price auction as the sealed-bid secondprice auction format in which only the losing bids are revealed. It is different from the English auction analyzed in Goeree (2000). In the latter the winner can still increase its bid after everybody else dropped out while in the modified second-prince format we exclude this possibility. All three types of auctions (simple- and modified second-price and English-auction) are different strategically ${ }^{4}$. Note that a separating equilibrium of

\footnotetext{
${ }^{4} \mathrm{~A}$ revenue equivalence result holds between the simple second-price auction and the English auction. See Goeree (2000).
} 
the modified second-price auction is also unique if it exists but it is not a full-signaling separating equilibrium because two different types can win and produce the same signal if the second largest bid was the same. In fact, the function $h(t)$ will be random because it will depend on the second largest bid, which is unknown to the winner in advance.

Theorem 1 Under $\boldsymbol{A 1}, \boldsymbol{A} 2$ and $\boldsymbol{A} 3$ the (full signaling) separating equilibrium of the first-price and the second-price auction will exist and yield the same revenue for the seller.

Proof. See Goeree $(2000)^{5}$.

In order to show that the separating equilibrium of the modified second-price auction yields different revenue than the separating equilibrium of the first- and of the (simple) second-price auction (Theorem 4) and to be able to characterize optimal auctions in the next section we make the following assumption, the assumption of additive separability:

Assumption 4 (A4): Assume that $V(t, h)=V_{1}(t)+V_{2}(h)$.

Assumption A4 is not the most compelling one because it states that an additional signaling possibility yields the same benefit for all of the types. This is against the intuitive ground of a traditional signaling set-up where signaling takes place because high types have more incentive for it than low types. In this model signaling will happen for another reason. A lower type will simply not follow a higher type because although it can buy a higher probability of winning, but doing so is not worthwhile since he will also have to pay more. So separation arises due to the same reason as in the standard auction case and the signaling feature of the outcome will arise only as a side effect. This additive separability property holds for example in our motivating story of the market with informed and uninformed consumers.

Because of the usual problems of signaling games we may have multiple equilibria. It is easy to show that there are many equilibria of both auction formats ${ }^{6}$. In order to

\footnotetext{
${ }^{5}$ After differentiating formula (3.6) and setting "the losing term" to zero in Goeree (2000), we can prove that only under $\mathbf{A 1}$ will the first-price auction have a separating equilibrium because the bid funtion is strictly increasing and the second-order condition is fulfilled. So despite revenue equivalence of the separating equilibria of the first-price auction and the simple second-price auction, there is an advantage of the first-price format, an interesting deviation from the standard case.

${ }^{6}$ For example, if the incumbent holds beliefs that any deviation of some finite number of bids signals that the lowest type has won, then it will not be rational to deviate from this finite number of bid levels so the beliefs of the incumbent will be self-enforcing, the usual problem of signaling games.
} 
reach a uniqueness result we introduce the following very strong, though not implausible assumption:

Assumption 5 (A5): Assume that the belief of the uninformed consumers about the expected value of the type of the winner, $h$ is weakly increasing in the winning bid in the first- and the simple second-price auctions. ${ }^{7}$

As it turns out A4 is not necessary for the desired uniqueness result so we introduce a weaker assumption instead of $\mathbf{A 4}$, the assumption of single-crossing:

Assumption (A-SC): The mixed partial derivative of $V(t, h)$ is non-negative

In the next lemma we state a uniqueness claim under assumptions:

Lemma 1 Under $\boldsymbol{A 1}, \boldsymbol{A} 2, \boldsymbol{A} 3, \boldsymbol{A}-\boldsymbol{S C}$ and $\boldsymbol{A 5}$, the only symmetric equilibrium of the first-price and the simple second-price auction is the (full-signaling) separating equilibrium.

Proof. We only prove the Lemma for the first price auction the result for the other format follows similarly. First we show that $t_{1}>t_{2}$ implies $p\left(t_{1}\right) \geq p\left(t_{2}\right)$ under singlecrossing. Assume the contrary, i.e. that $p\left(t_{1}\right)<p\left(t_{2}\right)$, which implies $b_{1}<b_{2}$ and this in turn by $\mathbf{A 5}$ implies $h_{1} \leq h_{2}$. Then we have the following incentive compatibility constraints for the two types:

$$
\begin{aligned}
& p\left(t_{1}\right)\left[V\left(t_{1}, h_{1}\right)-b_{1}\right] \geq p\left(t_{2}\right)\left[V\left(t_{1}, h_{2}\right)-b_{2}\right] \\
& p\left(t_{2}\right)\left[V\left(t_{2}, h_{2}\right)-b_{2}\right] \geq p\left(t_{1}\right)\left[V\left(t_{2}, h_{1}\right)-b_{1}\right]
\end{aligned}
$$

After adding these equations up we obtain:

$$
p\left(t_{1}\right)\left[V\left(t_{1}, h_{1}\right)-V\left(t_{2}, h_{1}\right)\right] \geq p\left(t_{2}\right)\left[V\left(t_{1}, h_{2}\right)-V\left(t_{2}, h_{2}\right)\right]
$$

Under single-crossing and by $p\left(t_{1}\right)<p\left(t_{2}\right)$ this can not happen, so $t_{1}>t_{2}$ must imply $p\left(t_{1}\right) \geq p\left(t_{2}\right)$. If $p\left(t_{1}\right)=p\left(t_{2}\right)$ then for all $t_{3} \in\left(t_{1}, t_{2}\right) p\left(t_{3}\right)=p\left(t_{1}\right)$ must hold by the previous step. This means that all the types on $\left(t_{1}, t_{2}\right)$ must have bid the same. This is not optimal though for these types because by raising the bid infinitesimally they can increase their chance of winning by a positive number and by $\mathbf{A} \mathbf{5}$ also $h$ would not decrease

\footnotetext{
${ }^{7}$ Note that this does not mean necessarily that the incumbent excludes the possibility that a higher type bids less than a lower; we only assume that a higher bid will induce a belief that on average the bidder is of higher type, i.e. $b_{2}>b_{1}$ implies $h_{2} \geq h_{1}$.
} 
if some of them do that deviation. So $p\left(t_{1}\right)>p\left(t_{2}\right)$ must hold which implies $b_{1}>b_{2}$ which clearly pins down a unique equilibrium with full signaling as in Goeree (2000).

We will analyze in the second part of this paper a function that will satisfy the strict version of A-SC (i.e. strict single-crossing), but for this first part mainly because of analytic tractability we will use additive separability. Now we turn to the comparison of the revenue generated by the simple and the modified second-price auctions under assumption A4 concentrating on the separating equilibria. First, a useful Lemma is given below:

Lemma 2 For any incentive compatible mechanism the winning probability, $p(t)$ is weakly increasing in $t$. Under $\boldsymbol{A} \mathbf{1}$ and $\boldsymbol{A} 4$, if two mechanisms yield the same equilibrium $p(t)$ function and they give the same utility for the lowest type, then every type will get the same utility in the two mechanisms' equilibria. In particular, independently of the function $h(t)$, if an equilibrium exists for a mechanism in which the probability of winning for type $t$ is $p(t)=F^{n-1}(t)$ and the lowest type gets zero utility, then in equilibrium this mechanism will give the same utility for every type $t$ as the first-price and the second-price auctions.

Proof. The expected utility of type $t$ if he pretends that his type is $\widetilde{t}$ is the following:

$$
U(t, \widetilde{t})=p(\widetilde{t}) V_{1}(t)+E_{z \mid \widetilde{t}}\left[p(\widetilde{t}) V_{2}(h(z))-b(\widetilde{t})\right]
$$

We may write the incentive compatibility constraints as follows:

$$
\begin{aligned}
& U(t, t) \geq U(t, \widetilde{t}) \\
& U(\widetilde{t}, \widetilde{t}) \geq U(\widetilde{t}, t)
\end{aligned}
$$

Add (IC1) to (IC2) using formula (6), cancel the terms that appear on both sides and get:

$$
[p(t)-p(\widetilde{t})]\left[V_{1}(t)-V_{1}(\widetilde{t})\right] \geq 0
$$

Using A1 we can conclude now that $p(t)$ is weakly increasing. 
Define $u(t)=U(t, t)$. By a similar argument to the standard case it follows that $u(t)$ is increasing and continuous, so almost everywhere differentiable. Using the envelope theorem we arrive at:

$$
u^{\prime}(t)=p(t) V_{1}^{\prime}(t)
$$

From (8) the conclusion follows, because the slope of $u(t)$ is the same for the two mechanisms and $u(\underline{t})$ is the same by assumption and we can use the Newton-Leibniz Theorem to conclude the result. The last result is a direct application of this more general one.

For later reference we define here welfare of bidder $i$ as the expected utility of bidder $i$ gross of his payment to the seller:

$W_{i}=E_{t}[u(t)+b(t)]$ for all $i$ with our symmetric mechanism. Define also total welfare as $W=\sum_{i} W_{i}{ }^{8}$

Also define the expected utility of agent $i$ as $E U_{i}=E_{t} u(t)$ for all $i$ for this symmetric mechanism.

Corollary 1 If both second price auction formats have a separating equilibrium, then the separating equilibrium of the modified second-price auction yields the same utility for every type $t$ as that of the (simple) second-price auction.

Proof. We have already seen in Lemma 2 that $p(t)$ is weakly increasing in equilibrium. But this means that the bids must be weakly increasing in this auction format. By the assumption that we have a separating equilibrium, we can rule out the flat parts of the bidding function. So the bids must be strictly increasing and then $p(t)=F^{n-1}(t)$ must hold. Then Lemma 1 applies because $p(\underline{t})=0$ and thus $U(\underline{t})=0$ for both second-price formats.

Note that $\mathbf{A 2}$ and $\mathbf{A 3}$ are not sufficient to ensure that a separating equilibrium of the modified second-price format exists. Define function $g(t)$ for the modified second-price auction as the function that satisfies:

$$
g(t)=E_{z \mid t}\left[V_{2}(h(z))\right]=E_{w \mid w \leq t}\left[V_{2}\left(E_{\tilde{t} \mid \tilde{t} \geq w}(\widetilde{t})\right)\right]
$$

\footnotetext{
${ }^{8}$ Note that $W$ can really be interpreted as total welfare since it is the sum of the (net) utilities of the bidders plus the revenue of the seller.
} 
If $t$ is the highest type then $p(t)=1$ and $z$ is the second highest type, thus $h(z)$ is the conditional expectation of the highest type if the second highest is $z$. If $t$ is not the highest then $p(t)=0$ and $h(z)$ does not matter. (This function, $g(t)$ can be interpreted as the expected value of $h(z)$ with respect to $z$ conditional on $t$ is winning.) Then using equation (6) we obtain the following equation under A4:

$$
U(t, \widetilde{t})=p(\widetilde{t})\left[V_{1}(t)+g(\widetilde{t})\right]-b(\widetilde{t})
$$

We can give a necessary and sufficient condition for the existence of a separating equilibrium of the modified second-price auction:

Lemma 3 Under $\boldsymbol{A} \mathbf{1}$ and $\boldsymbol{A}$, the separating equilibrium of the modified second-price auction exists if and only if $V_{1}(t)+g(t)+\frac{F(t) g^{\prime}(t)}{(n-1) f(t)}$ is strictly increasing in $t$.

The following lemma gives a similar condition for the simple second-price auction case:

Lemma 4 Under $\boldsymbol{A} 1$ and $\boldsymbol{A}$, the separating equilibrium of the (simple) second-price auction exists if and only if $V_{1}(t)+V_{2}(t)+\frac{F(t) V_{2}^{\prime}(t)}{(n-1) f(t)}$ is strictly increasing in $t$.

Proof. In the existence proof of Goeree (2000), he had to guarantee only that the bidding function is strictly increasing in $t$. In the simple second-price case this is true under the conditions of Lemma 4. The analysis of the modified second-price format is the same as the simple second-price auction, we only need to use $g(t)$ instead of $V_{2}(t)$ and proceed as in the simple case. This proves Lemma 3.

Theorem 2 Assume that the conditions of Lemma 3 and 4 hold and so the separating equilibria of the two second-price formats exist. Then the separating equilibrium of the modified second price auction yields strictly higher (smaller) revenue than that of the (simple) second-price auction if the function $V_{2}(t)$ is strictly concave (convex). If $V_{2}(t)$ is linear then the two formats yield the same amount of revenue.

Proof. Because of Corollary 1 the two formats provide the same utility level in equilibrium for every type $t$. So because of the quasi-linearity of the utility function of the bidders we need to compare only total welfare levels of the two auctions. If we write down the total welfare under the two mechanisms the difference will be just the following:

$$
n \Delta W=n\left(E_{t}\left[g(t) F(t)^{n-1}\right]-E_{t}\left[V_{2}(t) F(t)^{n-1}\right]\right) .
$$


Let $\widehat{t}$ be the second biggest type conditional on $t$ is the highest. Then the density function of $\widehat{t}$ is the following:

$$
\tilde{f}(\hat{t})=\frac{(n-1) F^{n-2}(\hat{t}) f(\hat{t})}{F^{n-1}(t)}
$$

First assume concavity. By concavity of $V_{2}$ :

$$
g(t)=E_{w \mid w \leq t}\left[V_{2}\left(E_{\widetilde{t} \mid \widetilde{t} \geq w}(\widetilde{t})\right)\right]>E_{w \mid w \leq t}\left[E_{\widetilde{t} \mid \widetilde{t} \geq w}\left(V_{2}(\widetilde{t})\right)\right]
$$

where $w$ stands for the type of the loser and $\widetilde{t}$ for the belief of the uninformed consumers about the type of the winner. Both $w$ and $\widetilde{t}$ has the same unconditional distribution as $t$. In the last formula, we could write strict inequality, because we assumed that $t$ has a non-degenerate distribution.

$$
g(t) F^{n-1}(t)>\int_{\underline{t}}^{t}(n-1) F^{n-2}(\hat{t}) f(\hat{t}) \int_{\widehat{t}}^{\bar{t}} \frac{f(v) V_{2}(v)}{1-F(\hat{t})} d v d \widehat{t}
$$

Now, using the last two formulas we may write the following inequality:

$$
\begin{aligned}
E\left(g(t) F^{n-1}(t)\right) & >\int_{\underline{t}}^{\bar{t}} \int_{\widehat{t}}^{\bar{t}} \int_{\widehat{t}}^{\bar{t}} \frac{(n-1) F^{n-2}(\hat{t}) f(\hat{t}) f(v) V_{2}(v) f(t)}{1-F(\hat{t})} d t d v d \widehat{t} \\
& =\int_{\underline{t}}^{\int_{t}} \int_{\widehat{t}}^{\bar{t}} f(v) V_{2}(v)(n-1) F^{n-2}(\hat{t}) f(\widehat{t}) d v d \widehat{t}
\end{aligned}
$$

After a change of the order of integration this implies:

$$
\begin{aligned}
E\left(g(t) F^{n-1}(t)\right) & >\int_{\underline{\underline{t}}}^{\bar{t}} \int_{\underline{\underline{t}}}^{v} f(v) V_{2}(v)(n-1) F^{n-2}(\widehat{t}) f(\widehat{t}) \hat{d t} d v \\
& =\int_{\underline{t}}^{\bar{t}} f(v) V_{2}(v) F^{n-1}(v) d v=E\left(V_{2}(t) F^{n-1}(t)\right)
\end{aligned}
$$

This last inequality yields the result for concavity. Convexity is handled similarly and the linear case follows easily along the same lines.

The previous Theorem shows that it is possible that withholding information may be beneficial for the seller. This question is analyzed in the next section in more detail. We need to take from this example only that it is not necessarily appropriate for the seller to completely exploit the signaling feature of the game. 


\subsection{Optimal mechanisms}

This section turns to the study of the mechanism design problem. The symmetric set-up of the previous section will be used, and we deal only with the two polar cases; i.e. the cases where $V$ is concave or convex in $h$. Note that concavity/convexity have their strength only because of the expected value interpretation of $h(z)$. We maintain the assumption of additive separability throughout. See our second example and the conclusion for the implications of dropping this assumption.

Another set of assumptions concerns the set of feasible mechanisms and the timing of the game. In this section the seller is able to commit to the mechanism, in particular he can commit not to collude with the winner ex post. The belief of the uninformed consumers is driven completely by the announced mechanism of the seller (and thus outof-equilibrium beliefs are inessential). These features are very useful because we can transform the problem at hand from a problematic signaling game (studied in the last section where ad-hoc assumptions concerning beliefs were used to reach uniqueness) to a fairly standard Bayesian mechanism design problem.

The use of asymmetric mechanisms is allowed but this will not change our results, because the optimal mechanism will be symmetric (as we show it later) in our symmetric environment. In this section bidders will be denoted by $1 \ldots . . n$. Let $t_{i}$ be the type of bidder $i$, and let $t=\left(t_{1}, . ., t_{n}\right)$ be the vector of types.

We turn now to the description of the timing in our situation. First the seller designs a mechanism, which includes the following ingredients ${ }^{9}$ :

1. Description of the winning probabilities of every type.

2. The expected value of payments for every type

3. $z$ is the (possibly random) signal sent to the uninformed consumers by the seller about the winning bidder after he reported $\widetilde{t}_{i} \cdot{ }^{10}$

In the next step the bidding firms announce their reports, $\widetilde{t}$. We assume that in

\footnotetext{
${ }^{9}$ See the revelation principle (e.g. Myerson (1979)), which allows us to concentrate on a direct mechanism.

${ }^{10}$ We assume that that whatever signal is sent to the consumers will be revealed also for the winner of the auction (and this is common knowledge) in order to avoid the complications of having to deal with the case where the consumers would also have private information. This assumption captures the idea that the signaling activity takes place to influence the belief of the consumers and so it is natural to assume that the winner will know the belief of the consumers for sure.
} 
equilibrium the bidders follow the pure strategies of always telling the truth even if they are indifferent between telling the truth and announcing a different type.

In the third step the seller determines who won (which can still be a stochastic variable) and announces the value of $z$ for the consumers and collects the payments of the bidders ${ }^{11}$. Based on the realization of the random variable $z$ the uninformed consumers can calculate the expected value $h_{i}(z)$ for every $z$.

Assuming that type $t_{i}$ reported $\widetilde{t}_{i}$ and the other reports were $\widetilde{t}_{-i}$ :

$$
U\left(t_{i}, \widetilde{t}_{i}, \widetilde{t}_{-i}, z\right)=p_{i}(\widetilde{t})\left[V_{1}\left(t_{i}\right)+V_{2}\left(h_{i}(z)\right)\right]-b_{i}\left(\widetilde{t}_{i}\right)
$$

Let

$$
u_{i}\left(t_{i}, t_{-i},, z\right)=u_{i}(t, z)=U\left(t_{i}, t_{i}, t_{-i}, z\right)
$$

Define the equilibrium utility of type $t_{i}$ of bidder $i$ as follows:

$$
u_{i}\left(t_{i}\right)=E_{z / t_{i}} u_{i}(t, z) .
$$

Define the winning probability of bidder $i$ if $\widetilde{t}_{i}$ was the announcement of the bidders to the seller, as follows:

$$
p_{i}\left(\widetilde{t}_{i}\right)=E_{t_{-i}} p_{i}\left(\widetilde{t}_{i}, t_{-i}\right)
$$

A condition that is parallel to Myerson (1981) will help us characterizing our optimal mechanism:

Assumption $6(\mathbf{A 6}): m\left(t_{i}\right)$ is increasing and positive for every $t_{i}$, where $m\left(t_{i}\right)=$ $V_{1}\left(t_{i}\right)-\frac{1-F\left(t_{i}\right)}{f\left(t_{i}\right)} V_{1}^{\prime}\left(t_{i}\right)$.

The payment of the bidders enters in the same quasi-linear way to the utility functions so only the expected value of the payments matter to both the bidders and the seller. In contrast, the randomization of the announcement matters because the bidders will be "risk averse" or "risk loving" in $h$ depending on whether we have concavity or convexity in $h$.

\footnotetext{
${ }^{11}$ Alternatively the seller himself could calculate $h_{i}(z)$ and announce this variable only. Under our assumptions this would make no difference and would completely eliminate the consumers from our model.
} 
Similarly to more standard set-ups, a "risk averse" agent will not like randomization but a "risk loving" will. This will be a very important property of our optimal mechanisms.

Because under $\mathbf{A} 4$ the $V_{2}$ component of the incentive compatibility constraints can be perfectly offset by the expected payment, it will not affect the $(I C)$ constraints. This implies that any (weakly) increasing $p_{i}\left(t_{i}\right)$ function can be implemented and we do not have to worry about the second order conditions. This feature will be made use of throughout this section.

\subsection{The concave case: "risk aversion"}

Assumption 7 (A7): Assume that $V$ is strictly concave in $h$.

A completely separating mechanism is one in whose equilibrium $p_{i}\left(t_{i}\right)=F^{n-1}\left(t_{i}\right)$ holds, i.e. always the highest type wins. Note that in the case of the first-price and secondprice auctions of the previous section a separating equilibrium is completely separating in our new definition.

Theorem 3 Assume that $V_{2}$ is non-negative for every possible value of the beliefs of the uninformed consumers. Then under $\boldsymbol{A 1}, \boldsymbol{A 2}, \boldsymbol{A 6}, \boldsymbol{A 7}$ the optimal mechanism for the seller is any no-signaling mechanism, in which always the bidder with the highest type wins. $^{12}$

Proof. Let us write first the expected utility of agent $i$ as follows:

$$
E U_{i}=\int_{\underline{t}}^{\bar{t}} f\left(t_{i}\right) u_{i}\left(t_{i}\right) d t_{i}
$$

After recalling the definition of welfare we have that the difference of total welfare and the sum of total (net expected) utilities gives $R$, the revenue of the seller:

$$
R=\sum_{i=1}^{n}\left[W_{i}-E U_{i}\right] .
$$

\footnotetext{
${ }^{12}$ E.g. a first-price auction with hiding the bids from the incumbent.
} 
In equilibrium welfare is given by the following expression:

$$
\sum_{i=1}^{n} W_{i}=\sum_{i=1}^{n}\left(\int_{\underline{t}}^{\bar{t}} p_{i}\left(t_{i}\right) f\left(t_{i}\right) V_{1}\left(t_{i}\right) d t_{i}\right)+E_{z}\left[V_{2}(h(z))\right]
$$

By a similar argument to the one in the proof of Lemma 2 we may write the equilibrium sum of utilities as follows:

$$
\begin{aligned}
T U & =\sum_{i=1}^{n} E U_{i}=\sum_{i=1}^{n} \int_{\underline{t}}^{\bar{t}} f\left(t_{i}\right) u_{i}\left(t_{i}\right) d t_{i}=\sum_{i=1}^{n} \int_{\underline{t}}^{\bar{t}} f\left(t_{i}\right) \int_{\underline{t}}^{t_{i}} p_{i}\left(v_{i}\right) V_{1}^{\prime}\left(t_{i}\right) d v_{i} d t_{i} \\
& =\sum_{i=1}^{n} \int_{\underline{t}}^{\bar{t}} p_{i}\left(t_{i}\right) V_{1}^{\prime}\left(t_{i}\right)\left(1-F\left(t_{i}\right)\right) d t_{i},
\end{aligned}
$$

where $u_{i}\left(t_{i}\right)$ is the utility achieved by bidder $i$ 's type $t_{i}$ in equilibrium. So,

$$
R=\sum_{i=1}^{n}\left(E_{t}\left[p_{i}\left(t_{i}\right)\left(V_{1}\left(t_{i}\right)-\frac{1-F\left(t_{i}\right)}{f\left(t_{i}\right)} V_{1}^{\prime}\left(t_{i}\right)\right)\right]\right)+E_{z}\left[V_{2}(h(z))\right] .
$$

By concavity of $V_{2}$ we have the following inequality:

$$
E_{z}\left[V_{2}(h(z))\right] \leq V_{2}\left(E_{t, z}\left[h(z) \mid t_{i} \text { winner }\right]\right)
$$

And we also know that:

$$
\begin{aligned}
E_{(t, z)}\left[\left(h(z) \mid t_{i} \text { winner }\right]\right. & =E_{(t, z)}\left\{E_{(x, y) \epsilon S\left(t_{i}, z i\right)}[x \mid(x, y) \text { winner }] \mid t_{i} \text { winner }\right\} \\
& =E_{t}\left[t_{i} \mid t_{i} \text { winner }\right]
\end{aligned}
$$

this last equation holds by the property of conditional expectation. So,

$$
E_{(t, z)}\left[V_{2}(h(z)) \mid t_{i} \text { winner }\right] \leq V_{2}\left\{E_{t}\left[t_{i} \mid t_{i} \text { winner }\right]\right\} \leq V_{2}\left\{E_{t}\left[t_{i} \mid t_{i} \text { is the highest }\right]\right.
$$

The last inequality holds because we sell the good with probability 1 in the optimal mechanism. ${ }^{13}$ From (28) it follows that

$$
R \leq \sum_{i=1}^{n}\left(E_{t}\left[p_{i}\left(t_{i}\right)\left(V_{1}\left(t_{i}\right)-\frac{1-F\left(t_{i}\right)}{f\left(t_{i}\right)} V_{1}^{\prime}\left(t_{i}\right)\right)\right]\right)+V_{2}\left(E_{t}\left[t_{i} \mid t_{i} \text { is the highest }\right]\right)
$$

\footnotetext{
${ }^{13}$ It seems possible to increase the expected value of $V_{2}$ conditional on selling the good by sometimes withholding the good. However, if we withhold the good then the $V_{2}$ component is not realized (i.e. we can take its value zero). We can prove that this loss from withholding the good exceeds the gain under concavity.
} 
Under A6 we can conclude (similarly to Myerson (1981)) that the first term is maximized if we sell the good to the highest type, so:

$$
R \leq \sum_{i=1}^{n}\left(E_{t}\left[F^{n-1}\left(t_{i}\right)\left(V_{1}\left(t_{i}\right)-\frac{1-F\left(t_{i}\right)}{f\left(t_{i}\right)} V_{1}^{\prime}\left(t_{i}\right)\right)\right]\right)+V_{2}\left(E_{t}\left[t_{i} \mid t_{i} \text { is the highest }\right]\right)
$$

Thus, we can conclude that a no-signaling, completely separating mechanism, which achieves the upper bound (given by the right hand side of the previous inequality) is optimal under concavity.

It easily follows from standard auction theory that under A1 and A4 this kind of mechanism exists, e.g. a first-price auction with hiding the bids from the uninformed consumers. ${ }^{14}$

A no-signaling, separating mechanism requires commitment on the part of the seller since he is fully aware of the type of the winner still he hides this information from the uninformed consumers. This is only possible under commitment because he would definitely have the incentive to reveal the type of the winner if this type is high and share the benefit with the winner. But this behavior would of course destroy the whole mechanism. If this commitment power is not assumed then the characterization of the optimal commitment under concavity is an open question. ${ }^{15}$

Theorem 3 characterizes the optimal mechanism in a very wide class of mechanisms. We will discuss in the Conclusion whether this mechanism is plausible and what happens if we change some assumptions concerning commitment possibilities and/or the functional form assumptions of $\mathbf{A} \mathbf{4}$ or the expected value interpretation of $h_{i}\left(t_{i}\right)$.

\subsection{The convex case: "risk loving bidders"}

Assumption 8 (A8): Assume that $V$ is strictly convex in $h$.

\footnotetext{
${ }^{14}$ Along the lines of footnote 9's proof we can show that we do not need to assume A2 or A3 for existence. Under A1 and A4 for any deterministic $h_{i}\left(t_{i}\right)$ structure in which $h_{i}\left(t_{i}\right)$ is weakly increasing, a separating equilibrium exists for the first-price auction (with the new belief function $h_{i}$ instead of full signaling). This separating equilibrium is of course a completely separating one. In a no-signaling mechanism $h_{i}\left(t_{i}\right)$ is constant so it is weakly increasing thus the equilibrium required in the Theorem exists, similarly for a full signaling mechanism (that is analyzed under convexity), where $h(t)=t$.

${ }^{15} \mathrm{~A}$ modified second-price auction may be an adequate mechanism if the seller has to announce all the information he has. (This auction can be organized as an ascending auction.)
} 
Remember that we call a mechanism deterministic if $h_{i}\left(t_{i}\right)$ does not have a random component and depends only on $t_{i}$ and not on the other reports.

Theorem 4 Assume that $V_{2}$ is non-negative for every possible value of the beliefs of the uninformed consumers. Then under $\boldsymbol{A 1}, \boldsymbol{A 4}, \boldsymbol{A 6}, \boldsymbol{A} 8$ the optimal deterministic mechanism for the seller is a full signaling, completely separating mechanism. ${ }^{16}$

Proof. We can proceed similarly to what we did in the concave case to show that the following holds for a deterministic mechanism:

$$
R=\sum_{i=1}^{n}\left(E_{t}\left[p_{i}\left(t_{i}\right)\left(V_{1}\left(t_{i}\right)-\frac{1-F\left(t_{i}\right)}{f\left(t_{i}\right)} V_{1}^{\prime}\left(t_{i}\right)\right)\right]\right)+E_{t}\left[V_{2}\left(h\left(t_{i}\right)\right) \mid t_{i} \text { is winner }\right]
$$

We will prove that the second term on the right hand side achieves its maximum value under full signaling. Assume that $t_{i}$ has the same signal as the set $T$ and so the same $h$. Denote this $h$ by $h(T)$. Switch now to the mechanism in which we report fully, so $h\left(t_{j}\right)=t_{j}$ holds for every type $t_{j}$ in $T$. Then by convexity of $V_{2}$ this step will increase the expectation of the term in question on this set $T$ without changing it outside of $T$. More formally:

$p\left(t_{j} \epsilon T \mid t_{j}\right.$ winner $) E_{t_{j} \epsilon T}\left[V_{2}\left(t_{j}\right) \mid t_{j}\right.$ winner $\left.\}\right]+p\left(t_{j} \epsilon T^{c} \mid t_{j}\right.$ winner $) E_{t_{j} \epsilon T^{c}}\left[V_{2}\left\{h\left(t_{j}\right) \mid t_{j}\right.\right.$ winner $\left.\}\right] \geq$ $p\left(t_{j} \epsilon T \mid t_{j}\right.$ winner $) V_{2}\left\{E_{t_{j} \epsilon T}\left[t_{j} \mid t_{j}\right.\right.$ winner $\left.]\right\}+p\left(t_{j} \epsilon T^{c} \mid t_{j}\right.$ winner $) E_{t_{j} \epsilon T^{c}}\left[V_{2}\left\{h\left(t_{j}\right) \mid t_{j}\right.\right.$ winner $\left.\}\right]=$ $p\left(t_{j} \epsilon T \mid t_{j}\right.$ winner $) V_{2}\{h(T)\}+p\left(t_{j} \epsilon T^{c} \mid t_{j}\right.$ winner $) E_{t_{j} \epsilon T^{c}}\left[V_{2}\left\{h\left(t_{j}\right) \mid t_{j}\right.\right.$ winner $\left.\}\right]$

$=E_{t}\left[V_{2}\left\{h\left(t_{j}\right) \mid t_{j}\right.\right.$ winner $\left.\}\right]$

where $T^{c}$ is the complement of $T$.

By a repeated application of this procedure until full signaling is achieved, we can still increase the term in question until there is any pooled reporting on any set $T$ that has positive measure. Using this last result we get:

$$
R \leq \sum_{i=1}^{n}\left(E_{t}\left[p_{i}\left(t_{i}\right)\left(V_{1}\left(t_{i}\right)-\frac{1-F\left(t_{i}\right)}{f\left(t_{i}\right)} V_{1}^{\prime}\left(t_{i}\right)\right)\right]\right)+E_{t}\left[V_{2}\left(t_{i}\right) \mid t_{i} \text { is winner }\right]
$$

Under A6 we can conclude (similarly to Myerson (1981)) that the first term is maximized if we always sell the good to the highest type, so:

$$
R \leq \sum_{i=1}^{n}\left(E_{t}\left[F^{n-1}\left(t_{i}\right)\left(V_{1}\left(t_{i}\right)-\frac{1-F\left(t_{i}\right)}{f\left(t_{i}\right)} V_{1}^{\prime}\left(t_{i}\right)\right)\right]\right)+E_{t}\left[V_{2}\left(t_{i}\right) \mid t_{i} \text { is winner }\right]
$$

\footnotetext{
${ }^{16}$ E.g. a first-price auction without hiding the bids from the incumbent.
} 
Because of monotonicity and the non-negativity of the function $V_{2}$, it is easy to see that the second conditional expectation on the right hand side of the last inequality is maximized if we sell always to the highest type. This yields:

$$
R \leq \sum_{i=1}^{n}\left(E_{t}\left[F^{n-1}\left(t_{i}\right)\left(V_{1}\left(t_{i}\right)-\frac{1-F\left(t_{i}\right)}{f\left(t_{i}\right)} V_{1}^{\prime}\left(t_{i}\right)\right)\right]\right)+E_{t}\left[V_{2}\left(t_{i}\right) \mid t_{i} \text { is the highest }\right]
$$

Thus, we can conclude that a full signaling, completely separating mechanism (the one that achieves the upper bound given by the right hand side expression) is optimal in the class of deterministic mechanisms under convexity.

Under A4, the selling problem can be considered in two steps: First maximize the expected signaling $V_{2}$ component conditional on winning for every probability structure, $p_{i}\left(t_{i}\right)$. Then taking this as constant we need to choose the probability structure, $p_{i}\left(t_{i}\right)$, that maximizes the first component of total revenue plus the value function that we got for the signaling component. Under convexity or concavity, complete separation (i.e. $\left.p(t)=F^{n-1}(t)\right)$ proves to be the optimal outcome but if we have a general $V_{2}$ function our proof does not go through. At this point we do not know whether the result goes through or not.

Theorem 4 is of less generality than Theorem 3 because it applies only to the class of deterministic mechanisms. If we stick to our interpretation that convexity amounts to risk loving" then one wonders whether randomization may improve welfare. In the proof of the following Proposition in the Appendix we will see an example for that possibility.

Proposition 1 Under $\boldsymbol{A} \mathbf{1}, \boldsymbol{A} 4, \boldsymbol{A 6}, \boldsymbol{A 8}$, the optimal symmetric mechanism is not necessarily deterministic.

Proof. See Appendix.

It is instructive to highlight the question of optimality of a random mechanism under convexity. It is certainly true that if we can find a random $h_{i}\left(t_{i}\right)$ structure, which is, expected value preserving in the sense that for every $t_{i}$

$$
E_{z \mid t}\left[h_{i}(z) \mid t_{i} \text { winner }\right]=t_{i}
$$

then convexity implies that

$$
E_{(t, z)}\left[V_{2}\left\{h_{i}(z)\right\} \mid t_{i} \text { winner }\right] \geq E_{(t, z)}\left[V_{2}\left(t_{i}\right) \mid t_{i} \text { winner }\right]
$$


so this mechanism would improve on the full-signaling separating one. It is not easy though to find a $h_{i}(z)$ structure that satisfies (36). In our example in the Appendix it does not hold either.

There is an additional and opposite effect. A random $h_{i}(z)$ on average tends to favor lower types in terms of the expected value of $h_{i}(z)$ which may be considered as a pooling feature that is not beneficial under convexity (under convexity the worst completely separating mechanism is the no-signaling one). The best example of these two opposite effects is our modified second-price auction where

$$
\begin{gathered}
E_{z \mid t}\left[h_{i}(z) \mid t_{i} \text { winner }\right] \geq t_{i} \text { for small } t_{i} \text { and } \\
E_{z \mid t}\left[h_{i}(z) \mid t_{i} \text { winner }\right] \leq t_{i} \text { for large } t_{i} .
\end{gathered}
$$

This is good under concavity but adverse under convexity. Of the two effects, the first was stronger in the sense that a modified second-price auction is better than a (simple) second-price format under concavity and worse in the convex case.

\section{Example 2}

\subsection{Model}

Assume that three firms are competing in a homogenous product market with the following demand function $P(Q)=a-Q$ where $P$ is the price and $Q$ is the industry output. Let the marginal cost of production constant, $c$ and the same for every firms.

The equilibrium profit of this initial three firms Cournot game is:

$$
V_{i}=\left(q_{i}\right)^{2}=\left(\frac{a-c}{4}\right)^{2}
$$

where $q_{i}$ is the quantity produced by firm $i$ and $c$ is the constant marginal cost.

Either firm one or two can merge with firm three but they can't merge to monopoly. Both combinations of firms can result in decreased marginal cost for the merged firm.

Suppose that firm one can decrease its marginal cost by $t_{1}$ and firm two by $t_{2}$. The cost savings are private informations and independently drawn from the interval $[0, c]$ according to the density $f_{i}$. Denote the distribution of $f_{i}$ by $F_{i}$ and suppose it is uniform 
and common knowledge. If firm $i$ decides to acquire firm three after the acquisition there will be two firms in the industry. The merged company with marginal cost of $c-t_{i}$ and the other firm with marginal cost $c$. So the profit of the combined firm can be calculated as $\pi_{i}\left(t_{1}\right)=\left(P-\left(c-t_{i}\right)\right) q_{i}$, while the profit of the third firm is $\pi_{j}\left(t_{i}\right)=(P-c) q_{j}$. We specify a two-stage game. First the seller designs a mechanism and then if the company is sold the two firms will play the post-auction Cournot game, in which the winner has cost advantage; if it is not sold then the Cournot game played is just the symmetric version, with marginal costs equal to $c$ for all three firms.

\subsection{The post auction Cournot game}

First describe the outcome of the post auction market game if merger occurred supposing that firm $w$ is the one that merged and firm $l$ is the other firm on the market. We will assume throughout that firm $l$ will produce positive quantity regardless of his belief about firm $w$. (We will give conditions for this to hold later.) We also assume that the losing firm does not have any private information (not known to the winner) in this stage. ${ }^{17}$ Then the winner will have the following maximization problem:

$$
\underset{q_{w}}{\operatorname{Max}}\left(a-q_{l}-q_{w}-\left(c-t_{w}\right)\right) q_{w}
$$

The first order condition yields:

$$
q_{w}=\frac{a-q_{l}-\left(c-t_{w}\right)}{2}
$$

The problem of the "losing firm" is a bit more complicated since it does not know the type of the winner. It has a belief which is coming from the uniform prior distribution and the information that was transmitted by the auction and the knowledge of its own type, $t_{j}$. Then firm $j$ will maximize its expected profit, and the distribution it is using is according to the belief it has:

$$
\max _{q_{l}} E_{l}\left(a-q_{l}-q_{w}-c\right) q_{l}
$$

\footnotetext{
${ }^{17}$ This means that whatever information is transmitted by the auction mechanism to the loser is also known by the winner and that the type of the loser is revealed to the winner (and this is common knowledge). These assumptions guarantee that the winner will know the posterior beliefs of the loser and thus he knows what the equlilibrium quantity of the loser will be. This restriction may be with loss of generality.
} 
The first order condition yields:

$$
q_{l}=\frac{a-c-E_{l} q_{w}}{2}
$$

Using (42) and (44) yields:

$$
q_{w}=\frac{a-c+2 t_{w}+E_{l} q_{w}}{4}
$$

If we take expectations of both sides of (45) after some algebra it yields:

$$
E_{l} q_{w}=\frac{a-c+2 E_{l} t_{w}}{3}
$$

We can rewrite (45) and (44) as:

$$
\begin{gathered}
q_{w}=\frac{a-c}{3}+\frac{t_{w}}{2}+\frac{E_{l} t_{w}}{6} \\
q_{l}=\frac{a-c}{3}-\frac{E_{l} t_{w}}{3}
\end{gathered}
$$

Note that by the time of the post auction Cournot game the beliefs of firm $l$ are predetermined, so $E_{l} q_{w}$ and $E_{l} t_{w}$ are given. From (47) and (48) we calculate the profits of the two firms as follows:

$$
\begin{gathered}
V_{\text {win }}=\left(a-q_{l}-q_{w}-\left(c-t_{w}\right)\right) q_{w}=\frac{(a-c)^{2}}{9}+\frac{t_{i}^{2}}{4}+\frac{\left\{E_{l} t_{w}\right\}^{2}}{36}+\frac{(a-c) t_{l}}{3}+\frac{(a-c) E_{l} t_{w}}{9}+\frac{t_{w} E_{l} t_{w}}{6} \\
V_{\text {lose }}=E_{l}\left(a-q_{l}-q_{w}-c\right) q_{l}=\frac{(a-c)^{2}}{9}-\frac{\left\{E_{l} t_{w}\right\}^{2}}{18}-\frac{(a-c) t_{w}}{6}-\frac{(a-c) E_{l} t_{w}}{18}+\frac{t_{w} E_{l} t_{w}}{6}
\end{gathered}
$$

It is a very important feature of this linear model that the loser's belief can be described by its expected value for our purposes. So, the information transmitted by the auction mechanism matters only because of its effect on the expected value of the winner's type from the loser's point of view.

\subsection{The auction process}

Now, we turn to the first stage of the game, i.e. the auction process. The main difference between this process and a standard auction is that here the seller can influence the beliefs of the loser and thereby also his own revenue by choosing how much information 
to announce about the winner. This additional feature is reflected in the third component of the mechanism below. The seller wants to maximize his revenue; in order to do that he designs a direct mechanism that includes the following ingredients ${ }^{18}$ :

1. Description of the winning probabilities of every type of every players. Let $p_{i}\left(\widetilde{t}_{i}, \widetilde{t}_{j}\right)$ be the probability that somebody with report $\widetilde{t}_{i}$ wins if his opponent reported $\widetilde{t}_{j}$.

2. The expected value of payments for every type.

3. The random signal $l_{i}\left(\widetilde{t}_{i}, \widetilde{t}_{j}\right)$, the seller sends to the players about bidder $i$ after he reported $\widetilde{t}_{i}$ and the loser reported $\widetilde{t}_{j}$. The seller can introduce additional noise so make $l_{i}$ a random variable even conditional on $\left(\widetilde{t}_{i}, \widetilde{t}_{j}\right)$. As it was mentioned already in Footnote 18 the message sent to the players is available for both players, so it is public.

In the next step the buyers announce their reports, $\widetilde{t}=\left(\widetilde{t}_{i}, \widetilde{t}_{j}\right)$. Assume that in equilibrium the bidders follow the pure strategies of always telling the truth even if they are indifferent between telling the truth and announcing a different type.

In the third step the seller announces who won and the realization of the random signal, $l_{i}\left(\widetilde{t}_{i}, \widetilde{t}_{j}\right)$ and collects the revenue from the bidders. After this auction phase the bidders play the Cournot game and finally they collect their payoffs.

Let's introduce the following piece of notation. Let the random variable, $h_{i}\left(t_{i}, t_{j}\right)$ denote the expected value of the winner's (player $i$ ) type from the loser's (player $j$ ) point of view after the auction if the winner's type was $t_{i}$ and the loser's type was $t_{j}$. We can calculate the belief of the loser conditional on having type $t_{j}$ and observing signal $l$ as follows:

$$
h_{i}\left(t_{i}, t_{j}\right)=E_{t_{i}}\left[t_{i} \mid\left\{t_{i} \text { is winner, } t_{j}, l\right\}\right]
$$

Note that this variable, $h_{i}\left(t_{i}, t_{j}\right)$ can be a random variable if the seller makes random announcements conditional on observing $\left(t_{i}, t_{j}\right)$. For this reason we will take expectation also with respect to $h$. (The previously used variable, $E_{l}\left(t_{w}\right)$ is just one particular realization of this $h$ variable.) Assume that the seller can credibly commit to the mechanism he designed so that he is directly able to determine the beliefs of the "loser" with respect to some constraints given later. In this way we can take the beliefs, $h_{i}\left(t_{i}, t_{j}\right)$ as the choice variable. We will follow this approach throughout this Section. We will concentrate on

\footnotetext{
${ }^{18}$ See the revelation principle (e.g. Myerson (1979)), which allows us to concentrate on a direct mechanism.
} 
symmetric mechanisms; we can do that without loss of generality, for this see MaskinRiley (1984) footnote 11. Let $V_{w i n, i}\left(t_{i}, h_{i}\left(t_{i}, t_{j}\right)\right)$ be the utility of type $i$ if he wins and $V_{\text {lose }, i}\left(t_{j}, h_{j}\left(t_{i}, t_{j}\right)\right)$ if he loses. (Note here that we have two bidders, $i$ not equal $j$, so the "losing utility" depends on the winner's type, like in Goeree (2000).)

So rewriting (49) and (50) with this new notation yields:

$$
\begin{gathered}
V_{w i n, i}\left(t_{i}, h_{i}\left(t_{i}, t_{j}\right)\right)=\frac{(a-c)^{2}}{9}+\frac{t_{i}^{2}}{4}+\frac{h_{i}^{2}\left(t_{i}, t_{j}\right)}{36}+\frac{(a-c) t_{i}}{3}+\frac{(a-c) h_{i}\left(t_{i}, t_{j}\right)}{9}+\frac{t_{i} h_{i}\left(t_{i}, t_{j}\right)}{6} \\
V_{\text {lose }, i}\left(t_{j}, h_{j}\left(t_{i}, t_{j}\right)\right)=\frac{(a-c)^{2}}{9}-\frac{h_{j}^{2}\left(t_{i}, t_{j}\right)}{18}-\frac{(a-c) t_{j}}{6}-\frac{(a-c) h_{j}\left(t_{i}, t_{j}\right)}{18}+\frac{t_{j} h_{j}\left(t_{i}, t_{j}\right)}{6}
\end{gathered}
$$

In the previous equation it was assumed (and indeed we will stick to this case throughout) that the loser will not exit from the market and produces a positive amount in every circumstances. The condition for this is that the "losing profit" will be positive even at the highest $t_{j}$ and $h_{j}$ (i.e. $h_{j}=t_{j}=c$ ). This profit is given by: $\frac{(a-2 c)^{2}}{9}$. This must be always positive. The quantity produced by the loser also need to be non-negative even at the highest $t_{j}$ and $h_{j}$ (i.e. $h_{j}=t_{j}=c$ ). The quantity is given by: $\frac{a-2 c}{3}$. So we need to require that $a \geq 2 c$.

\subsection{The bidders problem}

If bidder $i$ with type $t_{i}$ wins the auction and the expected value of his type according to the loser's belief is $h_{i}$ then he will have utility $V_{\text {win }, i}\left(t_{i}, h_{i}\right)$ in the Cournot game. If he loses against a type $t_{j}$ and his belief about the type of the winner is described by $h_{j}$ then his utility in the Cournot game is given by $V_{l o s e, i}\left(t_{j}, h_{j}\right)$. Finally if neither of the firms can buy the third then the equilibrium profit of firm $i$ is $\frac{(a-c)^{2}}{16}$. Players' overall utility must be decreased by the expected payment they make to the seller in the auction phase, $b_{i}{ }^{19}$

Thus the utility of type $t_{i}$ of player $\mathrm{i}$ if he reports $s_{i}$ is the following:

$$
\begin{aligned}
U_{i}\left(t_{i}, \widetilde{t}_{i}\right)=E_{t_{j}, h_{i}, h_{j}} & {\left[p_{i}\left(\widetilde{t}_{i}, t_{j}\right)\left(V_{\text {win }, i}\left(t_{i}, h_{i}\left(\widetilde{t}_{i}, t_{j}\right)\right)-b_{i}\left(\widetilde{t}_{i}, t_{j}\right)\right)\right.} \\
& \left.\left.+p_{j}\left(\widetilde{t}_{i}, t_{j}\right)\right) V_{\text {lose }, i}\left(t_{j}, h_{j}\left(\widetilde{t}_{i}, t_{j}\right)\right)+\left(1-p_{i}\left(\widetilde{t}_{i}, t_{j}\right)-p_{j}\left(\widetilde{t}_{i}, t_{j}\right)\right) \frac{(a-c)^{2}}{16}\right]
\end{aligned}
$$

\footnotetext{
${ }^{19}$ We assume here without loss of generality that only the wining bider pays.
} 
Bidder $i$ with type $t_{i}$ has to maximize $U_{i}\left(t_{i}, \widetilde{t}_{i}\right)$ with respect to $\widetilde{t}_{i}$; in equilibrium he must choose $\widetilde{t}_{i}=t_{i}$. The equilibrium utility of type $t_{i}$ is the following:

$$
\begin{aligned}
u_{i}\left(t_{i}\right)=U_{i}\left(t_{i}, t_{i}\right) & =E_{t_{j}, h_{i}, h_{j}}\left[p_{i}\left(t_{i}, t_{j}\right)\left(V_{w i n, i}\left(t_{i}, h_{i}\left(t_{i}, t_{j}\right)\right)-b_{i}\left(t_{i}, t_{j}\right)\right)\right. \\
+ & \left.\left.p_{j}\left(t_{i}, t_{j}\right)\right) V_{\text {lose }, i}\left(t_{j}, h_{j}\left(t_{i}, t_{j}\right)\right)+\left(1-p_{i}\left(t_{i}, t_{j}\right)-p_{j}\left(t_{i}, t_{j}\right)\right) \frac{(a-c)^{2}}{16}\right]
\end{aligned}
$$

It is easy to see that $u_{i}\left(t_{i}\right)$ is (weakly) increasing and continuous function, so it is almost everywhere differentiable. Then using the fact that $U_{i}\left(t_{i}, s_{i}\right)$ is maximized at $s_{i}=t_{i}$ the envelope theorem yields:

$$
u_{i}^{\prime}\left(t_{i}\right)=U_{i}^{(1)}\left(t_{i}, t_{i}\right)=E_{t_{j}, h_{i}}\left[p_{i}\left(t_{i}, t_{j}\right) V_{w i n, i}^{(1)}\left(t_{i}, h_{i}\left(t_{i}, t_{j}\right)\right)\right]
$$

where $U^{(1)}, V^{(1)}$ are the partial derivatives of $U, V$ with respect to the first variable.

Equation (56) follows from the (necessary) first order conditions for the problem of the bidders. As we will see this will determine the revenue of the seller directly without any reference to the sofar ignored second order conditions. To verify that we really have a maximum for the bidders at $\widetilde{t}_{i}=t_{i}$ we should check the (sufficient) global second order conditions. We will not characterize these conditions for a general mechanism instead we will prove at the end of the next Section that the auction that maximizes revenue will satisfy the second order conditions also, so it is really incentive compatible.

\section{The characterization of the optimal mechanism}

Lemma 5 For any symmetric, incentive compatible mechanism the revenue of the seller is given by the following expression:

$$
\begin{aligned}
R & =D+2 E_{\left(t_{1}, t_{2}, h_{1}\right)}\left[p _ { 1 } ( t _ { 1 } , t _ { 2 } ) \left\{\frac{5(a-c)^{2}}{144}-\frac{c(a-c)}{3}+\frac{3 t_{1}^{2}}{4}\right.\right. \\
& \left.\left.-\frac{h_{1}^{2}\left(t_{1}, t_{2}\right)}{36}+t_{1}\left(\frac{a}{2}-c\right)+\left(\frac{a-4 c}{18}\right) h_{1}\left(t_{1}, t_{2}\right)+\frac{t_{1} h_{1}\left(t_{1}, t_{2}\right)}{2}\right\}\right],
\end{aligned}
$$

where $D$ is a constant that does not depend on the mechanism. ${ }^{20}$

\section{Proof. See Appendix.}

\footnotetext{
${ }^{20}$ As we have already mentioned we can restrict attention to symmetric mechanisms without loss of generality.
} 
This result follows from our specific functional form assumptions after writing the revenue as the difference between Total Welfare and Total Utility of the bidders'. Upon using equation (56) to characterize the equilibrium utility of the different types (assuming an exogenous reservation utility) and calculating Total Welfare in the straightforward way we get the above result.

Corollary 2 The seller's problem, $(S P)$ can be written as below:

$$
\begin{gathered}
\max _{p_{1}, h_{1}} R=D+2 E_{\left(t_{1}, t_{2}, h_{1}\right)}\left[p _ { 1 } ( t _ { 1 } , t _ { 2 } ) \left\{\frac{5(a-c)^{2}}{144}-\frac{c(a-c)}{3}+\frac{3 t_{1}^{2}}{4}-\frac{h_{1}^{2}\left(t_{1}, t_{2}\right)}{36}\right.\right. \\
\left.\left.+t_{1}\left(\frac{a}{2}-c\right)+\left(\frac{a-4 c}{18}\right) h_{1}\left(t_{1}, t_{2}\right)+\frac{t_{1} h_{1}\left(t_{1}, t_{2}\right)}{2}\right\}\right] \\
\text { s.t. } 0 \leq p_{1}\left(t_{1}, t_{2}\right) \leq 1 \\
p_{1}\left(t_{1}, t_{2}\right)+p_{1}\left(t_{2}, t_{1}\right) \leq 1, \text { for all }\left(t_{1}, t_{2}\right) \\
0 \leq h_{1}\left(t_{1}, t_{2}\right) \leq c
\end{gathered}
$$

and consistency (we will analyze this constraint later) and global second order conditions for bidders' problems hold

Remark 1 In a symmetric mechanism $p_{2}\left(t_{1}, t_{2}\right)=p_{1}\left(t_{2}, t_{1}\right)$, so $p_{1}\left(t_{1}, t_{2}\right)+p_{1}\left(t_{2}, t_{1}\right) \leq 1$ must hold. Note that the first order conditions for bidders' maximization hold by construction, so we just need to check the second order conditions. Our consistency criterion for $h$ amounts to the requirement that there is a (possibly random) reporting policy of the seller such that the loser will hold beliefs as given by the function $h^{21}$

Define the expected value of $h$ conditional on the winner's type, $t_{w}$ as follows $\left(t_{l}\right.$ denotes the type of the loser):

$$
y\left(t_{w}\right)=E_{\left(t_{l}, h\right) \mid t_{w}} h\left(t_{w}, t_{l}\right)
$$

\footnotetext{
${ }^{21}$ The condition can be stated in the following form, assuming that 1 is the winner: There exists a (possibly random) reporting function, $l_{1}\left(t_{1}, t_{2}\right)$ such that the following holds for every pair, $\left(t_{1}, t_{2}\right)$ : $h_{1}\left(t_{1}, t_{2}\right)=E_{\left(t_{1}, l\right) \mid t_{2}}\left[t_{1} \mid t_{1}\right.$ is winner $]$
} 
and the probability of winning for a type, $t_{i}$

$$
p_{i}\left(t_{i}\right)=E_{t_{j}} p_{i}\left(t_{i}, t_{j}\right)
$$

Note that

$$
E_{t_{w}} y\left(t_{w}\right)=E_{t_{w}} E_{\left(t_{l}, h\right) \mid t_{w}} h\left(t_{w}, t_{l}\right)=E_{\left(t_{w}, t_{l}, h\right)} h\left(t_{w}, t_{l}\right)=E_{t_{w}} t_{w}
$$

where the last equality comes from the expected value interpretation of $h$ after applying the law of iterated expectations.

Lemma 6 For any symmetric, incentive compatible mechanism the following upper bound can be given for the revenue:

$$
R \leq D+2 E_{t} p(t)\left[\frac{5(a-c)^{2}}{144}-\frac{c(a-c)}{3}+\frac{3 t^{2}}{4}+\frac{5 a-11 c}{9} t-\frac{y(t)^{2}}{36}+\frac{t y(t)}{2}\right]
$$

Proof. See Appendix.

This last Lemma follows directly from the Jensen-inequality applied to a concave function. The intuition is that because the revenue is concave in $h$ the seller is better off if he does not randomize in his reporting policy. The bidders are better off (their total welfare is bigger) if no randomization occurs and so the seller can extract this extra utility from them by requiring to pay more. This result is similar in flavor to that of Maskin-Riley (1984), where they prove that under risk aversion (and some other technical conditions) a random mechanism is never optimal for the seller.

We have the following useful Lemma from Maskin-Riley (1984):

Lemma 7 (Maskin-Riley (1984)). Consider any symmetric mechanism. Let $p(t)$ the probability of winning for type $t$ as defined in (60). Then $p(t)$ must satisfy the following conditions:

$$
\begin{array}{ll}
\int_{x}^{c} p(t) d t \leq \int_{x}^{c} F(t) d t & \text { for every } x \in[0, c] . \\
0 \leq p(t) \leq 1 & \text { for every } t \in[0, c] .
\end{array}
$$

For any non-decreasing, piecewise differentiable $p(t)$ if these conditions hold then there exists a symmetric random rule that allocates the good with probability $p(t)$ to type $t$, i.e. $p(t)$ satisfies equation (60) for some symmetric mechanism $\left(p_{i}, h_{i}\right)$. 
Using our special distribution function, $F(t)=\frac{t}{c}$ we can write condition (63) for $p(t)$ to be a probability of allocation for any symmetric rule as follows: $\int_{x}^{c} p(t) d t \leq \int_{x}^{c} F(t) d t$ for every $x \in[0, c]$. Also $0 \leq p(t) \leq 1$ must be satisfied.

Lemma 8 The following three conditions hold for $y(t)$ if it is defined as in equation (59):

$$
\begin{aligned}
& \int_{x}^{c} p(t) y(t) d t \leq \int_{x}^{c} p(t) t d t \quad \text { for every } x \in(0, c] . \\
& \int_{0}^{c} p(t) y(t) d t=\int_{0}^{c} p(t) t d t . \\
& 0 \leq y(t) \leq c \quad \text { for every } t \in[0, c] .
\end{aligned}
$$

Proof. Because the random variable $h \in[0, c]$ and $y$ is just an expected value of $h$ this yields the third condition trivially. Consider the expression $\frac{\int_{x}^{c} p(t) t d t}{\int_{x}^{c} p(t) d t}$. Given our uniform prior distribution, this is just the expected value of the winner, conditional on that it is larger than $x$. Then $\frac{\int_{0}^{c} p(t) t d t}{\int_{0}^{c} p(t) d t}$ is just the expected value of the winner. Similarly $\frac{\int_{0}^{c} p(t) y(t) d t}{\int_{0}^{c} p(t) d t}$ is the expected value of $y(t)$ conditional on $t$ is winning. As we argued this is by the law of iterated expectations equals the expected value of the winner, hence the second condition follows. We now turn to the first condition. Imagine that winners that have type higher than $x$ are viewed are indistinguishable for the loser but they are distinguishable from winners below type $x$. Then by definition of $y(t)$ and after the use of the law of iterated expectations the first condition holds as an equality. Now suppose that the loser can not distinguish perfectly some types above $x$ and some types below $x$. Then the expectation of the loser regarding the winners type conditional on that this type is larger than $x$ will be smaller than the expected value of the winners type conditional on that it is larger than $x$, which is just the content of the first condition.

Let us introduce the notion of modified revenue. I will call the modified revenue of a mechanism, $\left(p_{i}\left(t_{i}, t_{j}\right), h_{i}\left(t_{i}, t_{j}\right)\right)$ the following expression:

$$
R^{m}=D+2 E_{t} p(t)\left[\frac{5(a-c)^{2}}{144}-\frac{c(a-c)}{3}+\frac{3 t^{2}}{4}+\frac{5 a-11 c}{9} t-\frac{y(t)^{2}}{36}+\frac{t y(t)}{2}\right]
$$

where $(p(t), y(t))$ are defined according to equations (60) and (59). So we can rewrite now our inequality (62) that holds for any given auction as follows:

$$
R \leq R^{m}
$$

Consider the following program, $\left(S P^{\prime}\right)$ : 


$$
\max _{\left(p_{i}\left(t_{i}, t_{j}\right), h_{i}\left(t_{i}, t_{j}\right)\right.} D+2 E_{t} p(t)\left[\frac{5(a-c)^{2}}{144}-\frac{c(a-c)}{3}+\frac{3 t^{2}}{4}+\frac{5 a-11 c}{9} t-\frac{y(t)^{2}}{36}+\frac{t y(t)}{2}\right]
$$

s.t.

$$
\begin{gathered}
\int_{x}^{c} p(t) y(t) d t \leq \int_{x}^{c} p(t) t d t \quad \text { for every } x \in(0, c] \\
\int_{0}^{c} p(t) y(t) d t=\int_{0}^{c} p(t) t d t \\
0 \leq y(t) \leq c \quad \text { for every } t \in[0, c] \\
\int_{x}^{c} p(t) d t \leq \int_{x}^{c} F(t) d t \text { for every } x \in[0, c] \\
0 \leq p(t) \leq 1 \quad \text { for every } t \in[0, c]
\end{gathered}
$$

$(p(t), y(t))$ are defined according to equations (60) and (59)

Then we have the following corollary of Lemma 7 and 8:

Corollary 3 Take any symmetric, incentive compatible mechanism $\left(p_{i}\left(t_{i}, t_{j}\right), h_{i}\left(t_{i}, t_{j}\right)\right)$. If $\left(p_{i}\left(t_{i}, t_{j}\right), h_{i}\left(t_{i}, t_{j}\right)\right)$ satisfies the constraints of program $(S P)$ then it also satisfies the constraints of program $\left(S P^{\prime}\right)$.

Lemma 9 Suppose that a symmetric mechanism $M^{*}$ satisfies the constraints of $(S P)$, it is an optimal solution to $\left(S P^{\prime}\right)$ and it is deterministic (i.e. $h_{i}$ depends only on $\left.t_{i}\right)$. Then this mechanism is optimal, i.e. it brings the highest revenue to the seller among all (incentive compatible) mechanisms.

Proof. Because $M^{*}$ satisfies $(S P)$ it is incentive compatible. Any other incentive compatible mechanism, $M$ satisfies the constraints of $(S P)$, so also that of $\left(S P^{\prime}\right)$. Then the modified revenue of $M^{*}$ is (weakly) larger than that of $M$, which is in turn (weakly) larger then the revenue of $M$. Because $M^{*}$ is deterministic it is easy to see that its 
revenue is the same as its modified revenue which is (weakly) larger than the revenue of any other symmetric, incentive compatible $M$. By our earlier remark we can concentrate on symmetric mechanisms without loss of generality, which concludes our proof.

We will show below that we can find a (deterministic) auction that satisfies the conditions of our lemma, so it is optimal. For this reason we now turn to program $\left(S P^{\prime}\right)$. We first state a useful Lemma:

Lemma 10 Suppose that a mechanism $M=\left(p_{i}\left(t_{i}, t_{j}\right), h_{i}\left(t_{i}, t_{j}\right)\right)$ is optimal solution to $\left(S P^{\prime}\right)$ and define $M^{\prime}=(p(t), y(t))$ as in equations (60) and (59). Then $(p(t), y(t))$ is also an optimal solution to $\left(S P^{\prime}\right)$. In searching for the optimal solution of (SP') we can restrict our attention to deterministic mechanisms.

Proof. By definition the objective function has the same value for $M$ and $M^{\prime}$. The constraints are clearly satisfied for $M^{\prime}$ if they hold for $M$. The second statement the trivially follows.

So instead of maximizing $R^{m}$ in the class of all (possibly) random it is enough to maximize with respect to all deterministic pairs, $(p(t), y(t))$. We obtain then the following program, $\left(S P^{\prime}\right)$ :

$$
\max _{(p(t), y(t))} D+2 E_{t} p(t)\left[\frac{5(a-c)^{2}}{144}-\frac{c(a-c)}{3}+\frac{3 t^{2}}{4}+\frac{5 a-11 c}{9} t-\frac{y(t)^{2}}{36}+\frac{t y(t)}{2}\right]
$$

s.t.

$$
\begin{gathered}
\int_{x}^{c} p(t) y(t) d t \leq \int_{x}^{c} p(t) t d t \quad \text { for every } x \in(0, c] \\
\int_{0}^{c} p(t) y(t) d t=\int_{0}^{c} p(t) t d t \\
0 \leq y(t) \leq c \quad \text { for every } t \in[0, c] \\
\int_{x}^{c} p(t) d t \leq \int_{x}^{c} F(t) d t \text { for every } x \in[0, c] \\
0 \leq p(t) \leq 1 \quad \text { for every } t \in[0, c]
\end{gathered}
$$


Theorem 5 (i) If $a \geq \frac{53}{5}$ c then the optimal solution of (SP") will involve $p(t)=F(t)$ and $y(t)=t$.

(ii) If $a<\frac{53}{5} c$ then there exists a positive $t_{0}$ such that the optimal solution of (SP") will involve $p(t)=0$ if $t<t_{0}$ and $p(t)=F(t), y(t)=t$ if $t \geq t_{0}$.

\section{Proof.}

$$
R^{m}=D+2 E_{t} p(t)\left[\frac{5(a-c)^{2}}{144}-\frac{c(a-c)}{3}+\frac{3 t^{2}}{4}+\frac{5 a-11 c}{9} t-\frac{y(t)^{2}}{36}+\frac{t y(t)}{2}\right],
$$

so basically we need to maximize:

$$
\begin{aligned}
& E_{t} p(t)\left[\frac{5(a-c)^{2}}{144}-\frac{c(a-c)}{3}+\frac{3 t^{2}}{4}+\frac{5 a-11 c}{9} t-\frac{y(t)^{2}}{36}+\frac{t y(t)}{2}\right] \\
& =\int_{0}^{c} \frac{p(t)\left(\frac{5(a-c)^{2}}{144}-\frac{c(a-c)}{3}+\frac{3 t^{2}}{4}-\frac{h^{2}(t)}{36}+\left(\frac{5 a-11 c}{9}\right) t+\frac{t q(t)}{2}\right)}{c} d t
\end{aligned}
$$

We want to formulate our problem as a Hamiltonian, so we first introduce

$$
\begin{gathered}
Z(x)=\int_{x}^{c} p(t) d t-\int_{x}^{c} F(t) d t \\
Y(x)=\int_{x}^{c} p(t) h(t) d t-\int_{x}^{c} p(t) t d t
\end{gathered}
$$

We will ignore the constraint that $0 \leq y(t) \leq c$ for every $t \in[0, c]$ and will see that it will be satisfied in the optimal solution of the relaxed program.

Then our problem is:

$$
\begin{gathered}
\max _{p(t), y(t)} \int_{0}^{c} p(t)\left(\frac{5(a-c)^{2}}{144}-\frac{c(a-c)}{3}+\frac{3 t^{2}}{4}-\frac{y^{2}(t)}{36}+\left(\frac{5 a-11 c}{9}\right) t+\frac{t y(t)}{2}\right) d t \\
\text { s.t. } Y(x) \leq 0 \quad \text { for every } x, \text { and } Y(c)=Y(0)=0 \\
Z(x) \leq 0 \quad \text { for every } x, \text { and } Z(c)=0
\end{gathered}
$$




$$
\begin{gathered}
Y^{\prime}(x)=p(x)(x-y(x)) \\
Z^{\prime}(x)=F(x)-p(x) \\
0 \leq p(t) \leq 1
\end{gathered}
$$

The Hamiltonian can be written as:

$$
\begin{aligned}
H & =p(t)\left(\frac{5(a-c)^{2}}{144}-\frac{c(a-c)}{3}+\frac{3 t^{2}}{4}-\frac{y^{2}(t)}{36}+\left(\frac{5 a-11 c}{9}\right) t+\frac{t y(t)}{2}\right) \\
& +\theta(t) p(t)(t-y(t))-\pi(t) Y(t)+\alpha(t)(F(t)-p(t))-\beta(t) Z(t)+w(t) p(t)+\psi(t)(1-p(t))
\end{aligned}
$$

Here the necessary conditions will be also sufficient because the objective function and the constraints are concave in the state and the control variables together. The necessary conditions are the following:

$$
\begin{aligned}
& \frac{\partial H}{\partial p}=\frac{5(a-c)^{2}}{144}-\frac{c(a-c)}{3}+\frac{27 t^{2}-y^{2}(t)}{36}+\frac{(5 a-11 c) t}{9}+\frac{t y(t)}{2}+w(t)-\psi(t)-\theta(t)(y(t)-t)-\alpha(t)=0 \\
& \frac{\partial H}{\partial y}=p(t)\left[\frac{t}{2}-\frac{y(t)}{18}-\theta(t)\right]=0 \\
& -\frac{\partial H}{\partial Y}=\pi(t)=\theta^{\prime}(t) \\
& -\frac{\partial H}{\partial Z}=\beta(t)=\alpha^{\prime}(t)
\end{aligned}
$$

$\beta, \pi, w, \psi \geq 0$ and $\beta, \pi, w, \psi=0$ if their constraints are not binding, $\theta, \alpha$ are piecewise differentiable and $\alpha(0) \geq 0$. (See more on this in the proof of Theorem 8 in Maskin-Riley (1984))

We propose a solution that will satisfy these necessary and sufficient conditions and then we're done. First assume that $a \geq \frac{53}{5} c$. Then we propose $p(t)=F(t), y(t)=t$ as a solution. This will surely satisfy all of our constraints and also possible; we just need to organize a first-price auction with announcing the type of the winner and pick the completely separating equilibrium of it. This equilibrium exists as it was shown in Goeree (2000). Because $0<p(t)<1$ for almost every type we have $\theta(t)=\frac{t}{2}-\frac{t}{18}=\frac{5 t}{9}$, $w(t)=\psi(t)=0$ and $\alpha(t)=\frac{5(a-c)^{2}}{144}-\frac{c(a-c)}{3}+\frac{11 t^{2}+t(5 a-11 c)}{9}$. We can prove that if $a \geq \frac{53}{5} c$ then $\alpha(0) \geq 0$ holds. Also $\pi(t)=\theta^{\prime}(t) \geq 0$ surely and $\beta(t)=\alpha^{\prime}(t)=\frac{22 t+(5 a-11 c)}{9} \geq 0$ under our assumptions. So we really have an optimal solution in this case. 
Now we turn to the case when $a<\frac{53}{5} c$. Let $t_{0}$ be the bigger solution of the following equation:

$$
A(t)=\frac{5(a-c)^{2}}{144}-\frac{c(a-c)}{3}+\frac{11 t^{2}+t(5 a-11 c)}{9}=0 .
$$

Under our assumptions $t_{0}>0$.

Then let $p(t)=0$ if $t \leq t_{0}$ otherwise let $p(t)=F(t)$. Let $y(t)=t$ for every $t$. Set $\theta(t)$ $=\frac{5 t_{0}}{9}$ and $\alpha(t)=0$ on $\left[0, t_{0}\right]$. If $t>t_{0}$ then $p(t)>0$ so $\theta(t)=\frac{5 t}{9}$ as before. In this range $\alpha(t)=\frac{5(a-c)^{2}}{144}-\frac{c(a-c)}{3}+\frac{11 t^{2}+t(5 a-11 c)}{9}$. Let $w(t)=-\left\{\frac{5(a-c)^{2}}{144}-\frac{c(a-c)}{3}+\frac{11 t^{2}+t(5 a-11 c)}{9}\right\}>0$ on $\left[0, t_{0}\right]$ and $w(t)=0$ if $t>t_{0}$. Let $\psi(t)=0$ for every $t$. Now, by construction $\frac{\partial H}{\partial h}=\frac{\partial H}{\partial p}=0$. Also, $\beta(t)=\alpha^{\prime}(t)=0$ on $\left[0, t_{0}\right)$ where the constraint (of which $\beta$ is the multiplier) does not bind and $\beta(t)=\alpha^{\prime}(t)>0$ if $t \geq t_{0} \cdot{ }^{22}$ Similarly, $\pi(t)=\theta^{\prime}(t)=0$ on $\left[0, t_{0}\right)$ and $\pi(t)=\theta^{\prime}(t)>0$ if $t>t_{0}$, and the constraint (of which $\pi$ is the Lagrange multiplier) always binds. We will have continuous multipliers so we don't have to worry about the jump conditions. All of the necessary (and here by concavity sufficient) conditions hold, so we found the optimal solution. The only difference between this second case and the first case is that our optimal full-signaling first-price auction now prescribes some positive reserve price.

Lemma 11 There exist an excepted payment schedule, $B(t)$ such that the mechanism $(p(t), y(t))$ given in the previous lemma if applied together with $B(t)$ then both the firstand the global second-order conditions for the bidders' problem will hold for every $t$.

Proof. As we have seen already there is a unique $B(t)$ such that the first order conditions hold if we use the mechanism $(p(t), y(t))$. We have to show thus only that the strategies of the bidders satisfy global second order conditions, if they satisfy the first order conditions. In the proposed mechanism $h\left(t_{i}\right)=t_{i}$ for all $t_{i}$. First take the case when we sell the good with probability 1, this happens if $a$ is big enough relative to $c$. In that case we know that $p\left(t_{i}\right)=\frac{t_{i}}{c}$ and the value of the expected payoff of type $t_{i}$, $B\left(t_{i}\right)$ is increasing, continuous in $t$. Then it is almost everywhere differentiable, so we

\footnotetext{
${ }^{22} \alpha^{\prime}(t)>0$ holds if $t>t_{0}$ because then $\alpha^{\prime}(t)=A^{\prime}(t)$ and $A(t)$ is a convex, quadratic function which has one positive root and one negative under our assumptions (this holds because the product of the two roots is $\frac{5(a-c)^{2}}{144}-\frac{c(a-c)}{3}$, which is negative here). This means that $A(t)$ must be increasing if it is above its larger root, i.e. $t_{0}$.
} 
may assume that it is actually everywhere differentiable. ${ }^{23}$ The utility of a type $t_{i}$ who reports $\tilde{t}_{i}$ (assuming that the other buyer reports truthfully) is as follows:

$$
U_{i}\left(t_{i}, \widetilde{t}_{i}\right)=p\left(\widetilde{t}_{i}\right) V_{w i n, i}\left(t_{i}, \widetilde{t}_{i}\right)+\left(1-p\left(\widetilde{t}_{i}\right)\right) E_{t_{j}>\widetilde{t}_{i}} V_{\text {lose }, i}\left(t_{j}, t_{j}\right)-B\left(\widetilde{t}_{i}\right)
$$

Take the partial derivative with respect to the second argument:

$$
U_{i}^{(2)}\left(t_{i}, \widetilde{t}_{i}\right)=p^{\prime}\left(\widetilde{t}_{i}\right) V_{w i n, i}\left(t_{i}, \widetilde{t}_{i}\right)+p\left(\widetilde{t_{i}}\right) V_{\text {win }, i}^{(2)}\left(t_{i}, \widetilde{t}_{i}\right)-p^{\prime}\left(\widetilde{t}_{i}\right) E_{t_{j}>\widetilde{t}_{i}} V_{l o s e, i}\left(t_{j}, t_{j}\right)-B^{\prime}\left(\widetilde{t}_{i}\right)
$$

We need to show for the global second order condition to hold, that this value is positive if $\widetilde{t}_{i}<t_{i}$ and negative if $\widetilde{t}_{i}>t_{i}$.

We also know from the first order condition for truthful reporting that:

$$
U_{i}^{(2)}\left(\widetilde{t}_{i}, \widetilde{t}_{i}\right)=0
$$

Upon substituting (77) to (76) we have:

$$
\begin{aligned}
U_{i}^{(2)}\left(t_{i}, \widetilde{t}_{i}\right) & =U_{i}^{(2)}\left(t_{i}, \widetilde{t}_{i}\right)-U_{i}^{(2)}\left(\widetilde{t}_{i}, \widetilde{t}_{i}\right) \\
& =p^{\prime}\left(\widetilde{t}_{i}\right)\left[V_{\text {win }, i}\left(t_{i}, \widetilde{t}_{i}\right)-V_{\text {win }, i}\left(\widetilde{t}_{i}, \widetilde{t}_{i}\right)\right]+p\left(\widetilde{t}_{i}\right)\left[V_{\text {win }, i}^{(2)}\left(t_{i}, \widetilde{t}_{i}\right)-V_{\text {win }, i}^{(2)}\left(\widetilde{t}_{i}, \widetilde{t}_{i}\right)\right]
\end{aligned}
$$

In the proposed mechanism $p^{\prime}\left(\widetilde{t}_{i}\right), p\left(\widetilde{t}_{i}\right)>0$. Also for the utility function given $t_{i}>\widetilde{t}_{i}$ implies that $V_{\text {win }, i}\left(t_{i}, \widetilde{t}_{i}\right)-V_{\text {win }, i}\left(\widetilde{t_{i}}, \widetilde{t}_{i}\right), V_{\text {win }, i}^{(2)}\left(t_{i}, \widetilde{t}_{i}\right)-V_{\text {win }, i}^{(2)}\left(\widetilde{t}_{i}, \widetilde{t}_{i}\right)>0$. Similarly $t_{i}<\widetilde{t}_{i}$ implies that $V_{\text {win,i }}\left(t_{i}, \widetilde{t}_{i}\right)-V_{\text {win }, i}\left(\widetilde{t}_{i}, \widetilde{t}_{i}\right), V_{\text {win }, i}^{(2)}\left(t_{i}, \widetilde{t}_{i}\right)-V_{\text {win }, i}^{(2)}\left(\widetilde{t}_{i}, \widetilde{t}_{i}\right)<0$. Putting these results together yields the conclusion that the global second order condition holds.

Now turn to the case when we retain the good with positive probability. If we sell the good to a type $t_{i}$ with positive probability then it is still the case that $p\left(t_{i}\right)=\frac{t_{i}}{c}$, but now there is a type $t^{*}$ such that $p\left(t_{i}\right)=0$ if $t_{i}<t^{*}$. For types $t \geq t^{*}$ the argument above goes through, so it clearly follows that types above $t^{*}$ do not want to mimic each other. Types below $t^{*}$ will have the same allocation so they do not want to mimic each other. Only two types of incentive constraints remained to check. First, that types below $t^{*}$ should not want to pretend to be above $t^{*}$. That follows, because of the following argument. Type $t^{*}$ is held on his reservation utility in equilibrium, which is equal to $u(0)$. Because we

\footnotetext{
${ }^{23}$ This is because it does not influence our revenues what happens on a set of types of measure zero.
} 
already argued that type $t^{*}$ does not want to pretend types strictly above $t^{*}$, he can not get a larger level of utility than $u(0)$ by pretending that he is above $t^{*}$. Thus for every $t>t^{*}, U_{i}\left(t^{*}, t\right) \leq u(0)$. But then monotonicity of $U_{i}$ in the first component implies that if $\widetilde{t}_{i}<t^{*}<t$, then $U_{i}\left(\widetilde{t}_{i}, t\right)<U_{i}\left(t^{*}, t\right) \leq u(0)$. This means that types below $t^{*}$ do not want to mimic type above $t^{*}$. Second, we need to show that types, $t$ above $t^{*}$ do not want to report below $t^{*}$. This is trivial, because types above $t^{*}$ will have a positive rent in equilibrium and by mimicking types below $t^{*}$ they would get only their reservation utility.

Corollary 4 The suggested mechanism satisfies all of the constraint of (SP), if we use our proposed $y(t)$ for the variable $h(t)$.

Proof. We have shown that the global second order conditions hold, and it is trivial that the other constraints of (SP) are satisfied expect the (vaguely stated) condition about $y(t)=h(t)$. But this constraint just means that there exists a reporting and allocating policy such $y(t)$ will be the belief of the loser concerning the type of the winner. But $y(t)=t$ in our proposed solution which is clearly possible, just follow the policy of perfect information revelation about the winner's type.

If we put together our results we obtain our main Theorem:

Theorem 6 The mechanism proposed in Theorem 5 is revenue maximizing for the seller.

Sofar we have assumed that the reservation utility was given exogenously but this is not so. The reason is that the utility achievable for a player that does not participate depends on what happens to the other player. We now show though that for the purpose of designing optimal mechanisms it was not a faulty assumption to take the reservation utility of the different types constant among types and mechanisms. For this we present the following argument. Not participating only means not bidding so $b(t)=p(t)=0$ but the utility still depends on the probability of the other player getting the good and his announced type. We want to achieve in our mechanism participation on the equilibrium path but we have to allow the bidders to choose not to participate. Formally we will just expand the strategy space by allowing to report $N$, i.e. not participating. Because on the equilibrium path everybody will participate the only effect of this new strategy will be is 
that it will determine the reservation utility of the players. It is clear thus that we have to punish the player the most severely possible if he chooses not participating. Because in this case $b(t)=p(t)=0$ we can only choose the other player allocation to minimize the non-participating agent's utility. Here we will make the consistency requirement that if a player is not participating the revealed type of the other player is correct on average. This minimization problem can not depend on the real type of non-participating agent because it is unknown, and it also does not depend on what happens if both players participate, so the reservation utility is constant for any type and possible mechanism. We have to minimize the following expression:

$$
\begin{aligned}
E_{t_{j}} V_{\text {lose }, i}\left(t_{j,} h_{j}\left(t_{j}\right)\right) & =E_{t_{j}}\left[p ( t _ { j } ) \left\{\frac{(a-c)^{2}}{9}-\frac{h_{j}^{2}\left(t_{j}\right)}{18}-\frac{(a-c) t_{j}}{6}\right.\right. \\
& \left.\left.-\frac{(a-c) h_{j}\left(t_{j}\right)}{18}+\frac{t_{j} h_{j}\left(t_{j}\right)}{6}\right\}+\left(1-p_{j}\left(t_{j}\right)\right) \frac{(a-c)^{2}}{16}\right]
\end{aligned}
$$

Note that the solution to this program has nothing to do with what we have done previously, since this characterizes the out-of-equilibrium "threats" of the mechanism while the previous solutions are relevant on the equilibrium path. Because we can not manipulate $t_{j}$, the type of the participating agent we just minimize with respect to $h_{j}\left(t_{j}\right)$ and $p_{j}\left(t_{j}\right)$. In this notation we used the fact that the signal $h_{j}$, can not depend on $t_{i}$, because $t_{i}$ is unknown. If the other player participates all the time (as it will on the equilibrium path) then $E_{t_{j}} h_{j}\left(t_{j}\right)=E_{t_{j}} t_{j}=\frac{c}{2}$, a constant. We will not solve here this problem but note that $y(t)=t$ and $p(t)$ as on the equilibrium path above (i.e. in the first case $p(t)=F(t)$ for every $t$, while in the second case $p(t)=F(t)$ only above $\left.t_{0}\right)$ satisfies the constraints of the program though it is not necessarily optimal. This means that we can punish the non participating agent at least as severely as if we just give him the allocation that is given to the lowest type agent in a first-price auction. To hold down the lowest type at this reservation utility level, which is given by the harshest possible punishment we have to let the lowest type player pay positive amount even though $p(t)=0$. This can be done by a first price auction with entry fee. Finally it is easy to see that we can not increase the revenue by designing a mechanism that induces non-participation on the equilibrium path. The reason for this is obvious; non-participation just means $p(t)=b(t)=0$ which was a possibility in our set of mechanism but did not turn out to be optimal. (Even in the second case where $p(t)=0$ held for low types we had an entry fee which obviously 
raises revenue.)

Finally consider what happens if we do not allow entry fee. We do not have a full analysis yet but our intuition is that it may worth to change the mechanism given above to one that holds down the utility of the lowest type by punishing him with non monetary means, i.e. by increasing the probability that the other player gets the good (this is only relevant in our second case) or change the signaling structure $h_{j}$ in order to minimize the payoff of the lowest type.

\subsection{Generalization}

We indicate some conditions under which we may achieve results in a more general case.

Let $V(\widetilde{t})$ denote the reserve value of the buyer, and $V(b)$ is the utility of the bidders if the good is not allocated and no payment is made (in our model these are both $\frac{(a-c)^{2}}{16}$ ). Define the following new variable ${ }^{24}$ :

$$
K\left(t_{1}, h_{1}\right)=V_{w i n, 1}\left(t_{1}, h_{1}\right)+V_{l o s e, 2}\left(t_{1}, h_{1}\right)-\frac{1-F\left(t_{1}\right)}{f\left(t_{1}\right)} V_{w i n, 1}^{(1)}\left(t_{1}, h_{1}\right)-V(s)-2 V(b)
$$

Let's denote the partial derivatives of $K\left(t_{1}, h_{1}\right)$ with respect to the $l$ th component $(l=$ $1,2)$ as $K^{(l)}\left(t_{1}, h_{1}\right)$. Define also

$$
N\left(t_{1}\right)=K\left(t_{1}, t_{1}\right)
$$

Assumption $9 N\left(t_{1}\right)$ is increasing in $t_{1}$ and positive for every $t_{1}$

Assumption $10 K\left(t_{1}, h_{1}\right)$ is concave in $h_{1}$

Assumption $11 K^{(2)}\left(t_{1}, t_{1}\right)$ is increasing in $t_{1}$

Theorem 7 Under these three assumptions the optimal mechanism is to set $p(t)=F(t)$ and $h(t)=t$ for every $t$.

Proof. Because of assumption (10) we can prove again that the modified revenue is (weakly) larger then the real revenue. So we can maximize the modified revenue again

\footnotetext{
${ }^{24}$ For the see significance of this variable see equation (112) in the Appendix applied to a symmetric mechanism.
} 
with the same kinds of constraints and if we arrive at a deterministic optimum then we are done. We have to change the following constraints to allow for general distributions:

$$
\begin{gathered}
Z(x)=\int_{x}^{c} f(t) p(t) d t-\int_{x}^{c} f(t) F(t) d t \\
Y(x)=\int_{x}^{c} p(t) f(t) h(t) d t-\int_{x}^{c} f(t) p(t) t d t
\end{gathered}
$$

Thus,

$$
\begin{aligned}
& Y^{\prime}(x)=p(x) f(x)(x-h(x)) \\
& Z^{\prime}(x)=f(x)(F(x)-p(x))
\end{aligned}
$$

Then our problem is (the counterpart of (SP") the following ${ }^{25}$ :

$$
\max _{p(t), h(t)} \int_{0}^{c} p(t) f(t) K(t, h) d t
$$

$$
\begin{aligned}
\text { s.t. } Y(x) & \leq 0 \quad \text { for every } x \\
Y(c) & =Y(0)=0 \\
Z(c) & =0, Z(x) \leq 0 \quad \text { for every } x \\
Y^{\prime}(x) & =p(x) f(x)(x-h(x)) \\
Z^{\prime}(x) & =f(x)(F(x)-p(x)) \\
0 & \leq p(t) \leq 1
\end{aligned}
$$

If we follow the same method as we did in the Cournot-example (but of course we have to use a general density function, $f$ ) then we obtain the following Hamiltonian:

$$
\begin{aligned}
& H=p(t) f(t) K(t, h)+\theta(t) p(t) f(t)(t-h(t))-\pi(t) Y(t)+ \\
& \quad \alpha(t) f(t)(F(t)-p(t))-\beta(t) Z(t)+w(t) f(t) p(t)+\psi(t) f(t)(1-p(t))
\end{aligned}
$$

\footnotetext{
${ }^{25}$ See equation (112) in the Appendix, where we characterized the rvenue of an arbitrary mechanism. We need to apply this formula to a symmetric mechanism and also to use the (naturaly) generalized definition of modified revenue in this more general context to see that this program is really just the general version of (SP)".
} 
Using assumption (10) again we can see that the necessary conditions of the maximization will be also sufficient. We have the following necessary conditions:

$$
\begin{gathered}
\frac{\partial H}{\partial q}=p(t)\left[K^{(2)}(t, h)-\theta(t)\right]=0 \\
\frac{\partial H}{\partial p}=K(t, h)+w(t)-\psi(t)-\theta(t)(h(t)-t)-\alpha(t)=0 \\
-\frac{\partial H}{\partial Y}=\pi(t)=\theta^{\prime}(t) \\
-\frac{\partial H}{\partial Z}=\beta(t)=\alpha^{\prime}(t)
\end{gathered}
$$

$\beta, \pi, w, \psi \geq 0$ and $\beta, \pi, w, \psi=0$ if their constraints are not binding, $\theta, \alpha$ are piecewise differentiable and $\alpha(0) \geq 0$.

Now, in the proposed solution $\theta(t)=K^{(2)}(t, t)$, which is increasing by assumption (11). It is also differentiable because $V_{\text {win }}$ and $V_{\text {lose }}$ are differentiable by assumption. Thus $\theta^{\prime}(t)$ exists and positive for all $t$. Also by (10) (setting $w=0$ and $\psi=0$ because their constraints are not binding) $\alpha(t)=K(t, t)$ which is also increasing, positive by assumption (9) and differentiable. So, $\alpha^{\prime}(t)>0$ for all $t$. Finally we can note that the constraint of $Z$ and $Y$ are always binding so $\beta, \pi>0$ is not a problem.

Remark 2 Consider the set-up of the first model. For this, we assume $V_{\text {lose }}=V(s)=$ $V(b)=0$ and additive separability, i.e. $V_{\text {win }}(t, h)=V_{1}(t)+V_{2}(h)$. Then assumption (10) and (11) contradicts to each other. To see that we note that $K^{(2)}(t, t)=V_{2}^{\prime}(t)$. Assumption (11) amounts then to the convexity of $V_{2}$. The second derivative of $K(t, h)$ with respect to $h$ must be negative for all $h$ by assumption (10). But this second derivative is just $V_{2}^{\prime \prime}(h)$ and this should be positive for every value of the argument because $V_{2}$ is convex by assumption (11). The Cournot example avoids this problem by having a single-crossing functional form (it has positive mixed partial derivative). Indeed the Cournot example satisfies all three assumptions with the possible exception of the positivity in assumption $(9)$. 


\section{Conclusion}

In this concluding section, the role and acceptability of some of our major assumptions and the paths for future research will be discussed. First we assess how strong our assumptions about the commitment power of the seller are and which of the results would change upon relaxing them. The seller can both credibly transmit information and can prevent information transmission if it is in his best interest. The implementation of our optimal mechanism under concavity in Example 1 relies on the second assumption. Upon relaxing this assumption, pooling would be more appealing for the seller because the seller can credibly commit not to release the information only if he does not acquire it. As it was already noted, in Example 1 a pooling mechanism may become optimal under strong concavity and/or if the $V_{1}$ component is much less important than the $V_{2}$ one. Under convexity in Example 1 the optimal deterministic mechanism would still work without any of this commitment power of the seller (i.e. the capability to send and hide signals credibly). First, the full-information is revealed, so hiding information is not necessary. Second, if we organize this optimal mechanism as a first-price auction without hiding the winner's bid (this is possible, see footnote 19) then the auction process itself will transmit the information. This information transmission mechanism will not depend on the seller if the administration of the auction can be controlled, a not too strong assumption. In our Example 2 the optimal mechanism involves no hiding on information from the loser and it can be organized through a first-price auction, thus the commitment problem is basically absent. Along these lines, the following question is raised naturally: What is the optimal mechanism in Example 1 under concavity if information transmission is based exactly on the set of all the publicly observable variables, for example bids in a first-price auction? 26

The case where signaling is harmful, (i.e. the case of the Bertrand competition in the post-auction market) can be dealt with under additive separability by substituting $\left(-V_{2}\right)$ for $V_{2}$. Das Varma (2000) and Goeree (2000) note that in a first-price auction (with full

\footnotetext{
${ }^{26}$ Beyond the commitment problems above the seller and the winning bidder may have incentives to collude, i.e. to report higher type and share the benefit of this cheating. We exclude this possibility; otherwise it is easy to see that always the highest type is reported.If the transfer between the seller and the buyer is verifiable to third parties then this problem is much less severe, but not completely eliminated. We can imagine though that the procurement process is controlled by outside observers which makes commitment possible.
} 
announcement of the bids) a separating equilibrium does not necessarily exist. However even if existence problems do not arise, the results would change because if we switch to complete separation then the second expectation in (27) will be decreasing and not increasing. In general if the $\left(-V_{2}\right)$ component is strong than instead of $p(t)=F(t)$ other $p_{i}\left(t_{i}\right)$ structure may be optimal, maybe even a completely flat $p_{i}\left(t_{i}\right)$ function. However, if the $V_{1}$ component dominates then $p(t)=F(t)$ (if possible) may retain optimality.

The most restrictive point of the paper is the interpretation of the signaling variable $h$. Two kinds of generalizations are possible. The first maintains the assumption that $h$ is a given vector-function but drops the expected value interpretation and considers a more general class of vector valued variables. If we assume for example that not only the expected value (the first moment of the distribution) matters but also higher moments and if we assume also some appropriate functional form (e.g. convexity or concavity) then our results in Example 1 still go through. A problem with this approach is that it is even less clear what it means that utility is concave in the second moment of the distribution. The other possibility is to abandon the reduced form considerations for $h$ and see what the interesting signaling variables are in economically relevant situations. We followed this line in our Example 2, where we gave an example where only the expected value matters but there is a story behind the whole problem. It is easy to see that if we have the same model except that with quadratic cost function then our approach is still applicable. But generalization is extremely difficult to other cost or demand conditions since for example if we assume cubic cost function then it is not even possible to write the profit of the firms as a function that involves only moments of some distribution or any other easily treatable variables.

\section{Appendix}

Proof of Proposition 1:

Proof. We will give here an example where the full signaling, completely separating mechanism yields lower revenue than a random mechanism given below. For this consider the easiest setting. Take $n=2$ and let be the types uniformly distributed on the unit interval. In this section we will use only symmetric mechanisms so we will omit the $i$ subscripts everywhere and use of the notation of section where $t$ and $z$ were real numbers 
and not vectors. Consider the following random mechanism! If a type $t$ is between $y$ and $(1-y)$ then use a reporting function $l(t, z)=(t+z)$ where $z$ is uniform on $(-y, y)$ and independent of $t$. If a type $t$ is at the extremes then let's use $l(t)=t$ surely. We take $V_{2}(h)=h^{a}$ and finally let $p(t)=F(t)$, so always the higher type wins. We will prove that if we let $y$ to be small and the integer $a(>1)$ to be large enough then this random mechanism gives higher revenue than the full-signaling, completely separating mechanism.

We need to compare only the level of total welfare again because both mechanisms are completely separating and so utilities are the same for every type. First we note that both mechanisms give the same expected value of the first term in total welfare, equation (23) so we need to analyze only the behavior of the second term in (23). The optimal deterministic mechanism yields the following expression for the second expectation after substituting $h(t)=t$ and $n=2$ in equation (23):

$$
E_{t}\left[V_{2}(t) \mid t \text { winner }\right]=E_{t}\left[V_{2}(t) F(t)\right]=E_{t}\left[t^{a} F(t)\right]=E_{t}\left[t^{a} \mid t \text { winner }\right] .
$$

We calculate now the appropriate value for our suggested random mechanism. First let's introduce our usual function $g(t)$ as follows:

$$
p(t) g(t)=E_{z / t}[p(t, z) V 2(h(t, z)) \mid(t, z) \text { winner }]
$$

where the expectation is taken according to the conditional distribution of $z$ given $t$ and that $(t, z)$ is winner. But in our case this function simplifies to $g(t)=E_{z \mid t}\left[V_{2}(h(t, z)]\right.$ where the expectation is taken according to the conditional distribution of $z$ given $t$. Now the second term in equation (23) can be written as:

$$
E_{t}\left[V_{2}(t) \mid t \text { is winner in this random mechanism }\right]=E_{t} g(t) F(t)
$$

We must concentrate thus on the sign of the following expression:

$$
E_{t}\left[\left\{g(t)-t^{a}\right\} F(t)\right]=\int_{0}^{1}\left(g(t)-t^{a}\right) f(t) F(t) d t
$$

First we note a useful property of $l(t, z)$ : 
Lemma 12 Define $k(t, z)=E_{l(x, w)=l(t, z)}[x]$. If $l(t, z) \leq(1-2 y)$ then $k(t, z) \geq l(t, z)$. Moreover if $2 y \leq l(t, z) \leq(1-2 y)$ then $k(t, z)=l(t, z)$.

Proof. We prove the Lemma in two parts.

Part 1: If $y<l(t, z)<2 y$ then $k(t, z) \geq l(t, z)$.

If $2 y \leq l(t, z) \leq(1-2 y)$ then $k(t, z)=l(t, z)$. First note that $k(t, z)=E_{(x, w) s . t . l(x, w)=l(t, z)}[x]$. By assumption $w$ has the same distribution as $z$ and is independent of $x$. Also in both ranges of $l(t, z)$ it is equal to $t+z$ and thus $l(x, w)$ being in the same range we have $l(x, w)=x+w$. Using this we can rewrite $k(t, z)$ in both ranges of $l(t, z)$ as follows:

$$
k(t, z)=E_{(x, w) \text { s.t }} x=t+z-w[x]
$$

a, If $y<l(t, z)=t+z \leq 2 y$ then the last expectation is the same as:

$$
\begin{aligned}
k(t, z) & =E_{w}[(t+z-w) \mid(t+z-w)=y]=E_{w}[(t+z-w)] \\
& =(t+z)-E_{w}[w]=(t+z)=l(t, z)
\end{aligned}
$$

$\mathbf{b}$, If $2 y \leq l(t, z) \leq(1-2 y)$ then $k(t, z)=E_{w}[(t+z-w)]=l(t, z)$, because here we do not need to worry about any conditioning. Q.E.D.

Part 2: If $l(t, z)<y$ then $k(t, z) \geq l(t, z)$.

Proof of Part 2.: Note that if $l(t, z)<y$ is reported than only a type that is exactly $l(t, z)$ or a type that is bigger than $l(t, z)$ could be the winner. But then the definition of $k(t, z)$ yields the result. Q.E.D.

We now turn to the proof of Proposition 1:

We write the integral of equation (95) in the following form:

$$
\begin{aligned}
n d W & =I_{1}+I_{2}+I_{3} \\
& =\int_{0}^{3 y}\left(g(t)-t^{a}\right) \mathrm{f}(t) \mathrm{F}(t) \mathrm{dt}+\int_{3 y}^{1-3 y}\left(g(t)-t^{a}\right) \mathrm{f}(t) \mathrm{F}(t) \mathrm{dt}+\int_{1-3 y}^{1}\left(g(t)-t^{a}\right) \mathrm{f}(t) \mathrm{F}(t) \mathrm{dt} .
\end{aligned}
$$

Let's consider three cases according to the realization of $t$ : 
a, $t<3 y$ :

First note that $l(t, z) \leq 4 y<(1-2 y)$ (for small $y$ ) so Lemma 12 applies and $k(t, z) \geq$ $l(t, z)$. Because $a>1$ the function $V_{2}$ is convex so

$$
\begin{gathered}
g(t)=E_{z \mid t}\left[V_{2}(h(t, z)] \geq V_{2}\left(E_{z \mid t}[h(t, z)]\right)=V_{2}\left\{E_{z \mid t} E_{l(x, w)=l(t, z)}[x \mid(x, w) \text { winner }]\right\}=\right. \\
=V_{2}\left\{E_{z \mid t} E_{l(x, w)=l(t, z)}[x \mid x \text { is the highest }]\right\} \geq V_{2}\left\{E_{z \mid t} E_{l(x, w)=l(t, z)}[x]\right\}=V_{2}\left\{E_{z \mid t} k(t, z)\right\} \\
\geq V_{2}\left\{E_{z \mid t} l(t, z)\right\}=V_{2}(t)=t^{a}
\end{gathered}
$$

where the third to last step follows from Lemma 12. So,

$$
I_{1} \geq 0
$$

b, $3 y \leq t \leq(1-3 y)$ :

On this interval:

$$
h(t, z)]=E_{l(x, w)=l(t, z)}[x \mid(x, w) \text { winner }] \geq E_{l(x, w)=l(t, z)}[x]=k(t, z)=l(t, z)
$$

where the last step follows from Lemma 12 using the fact that $2 y \leq l(t, z) \leq(1-2 y)$ and thus $l(t, z)=k(t, z)$.So,

$$
g(t)=E_{z \mid t}\left[V_{2}\{h(t, z)\}\right] \geq E_{z \mid t}\left[V_{2}\{l(t, z)\}\right]=\int_{t-y}^{t+y} \frac{u^{a}}{2 y} d u
$$

Calculating the last integral and using inequality (102) yields:

$$
g(t) \geq t^{a}+y^{2} a(a-1) / 6+\text { higher order terms in } y(\text { or h.o.t. }) .
$$

Using inequality (103) we can rewrite $I_{2}$ as follows:

$$
I_{2}=\int_{3 y}^{1-3 y}\left(g(t)-t^{a}\right) F(t) f(t) d t \geq \int_{3 y}^{1-3 y} \frac{y^{2} a(a-1)}{6} d t+\text { h.o.t. }=\frac{y^{2} a(a-1)}{12}+\text { h.o.t. }
$$

So, we got a lower bound for the gain on this interval from switching from the optimal deterministic mechanism to our proposed random one. 
$\mathbf{c}, t>(1-3 y)$ :

First estimate $g(t)$ on this interval.

$$
g(t)=E_{z \mid t}\left[V_{2}\{h(t, z)\}\right] \geq V_{2}\left(E_{z \mid t}[h(t, z)]\right) \geq V_{2}(t-2 y),
$$

because $h(t, z) \geq(t-2 y)$ for every $z$. (This is trivial since a type $t$ is never reported to have the same signal as a type below $t-2 y$, because of the construction of the random variable z.) Using inequality (105) we get that for every $t$ in this interval the following holds:

$$
g(t)-V_{2}(t) \geq V_{2}(t-2 y)-V_{2}(t)=(t-2 y)^{a}-t^{a}=-2 y a t^{(a-1)}+\text { h.o.t. } \geq-2 y a+\text { h.o.t. }
$$

Thus from inequality (106) it follows that for $I_{3}$ the following holds:

$$
I_{3}=\int_{1-3 y}^{1}\left(g(t)-t^{a}\right) F(t) f(t) d t \geq(-2 y a+\text { h.o.t. }) \int_{1-3 y}^{1} t d t=-6 a y^{2}+\text { h.o.t. }
$$

We have from formulas (100), (104) and (107) after substitution:

$$
n \Delta W=I_{1}+I_{2}+I_{3} \geq 0+\left[\frac{y^{2} a(a-1)}{12}\right]-6 y^{2} a+\text { h.o.t. }
$$

Now if $y$ is small then the higher order terms will not matter only the coefficient of $y^{2}$. If $a>73$ holds then the coefficient of $y^{2}$ in $n \Delta W$ will be positive and the sign of the whole expression will be positive for a small enough $y$.

The proof of Lemma 5 .

Proof. Let $R^{i}$ be the expected revenue from player $i$. After using (40) we obtain the following expression for $R^{1}$ :

$$
\begin{aligned}
R^{1} & =E_{\left(t_{1}, t_{2}, q_{1}, q_{2}\right)} b_{1}\left(t_{1}, t_{2}\right)=E_{\left(t_{1}, t_{2}, q_{1}, q_{2}\right)}\left[p_{1}\left(t_{1}, t_{2}\right) V_{w i n, 1}\left(t_{1}, q_{1}\left(t_{1}, t_{2}\right)\right)\right. \\
& \left.+p_{2}\left(t_{1}, t_{2}\right) V_{\text {lose }, 1}\left(t_{2}, q_{2}\left(t_{1}, t_{2}\right)\right)+\left(1-p_{1}\left(t_{1}, t_{2}\right)-p_{2}\left(t_{1}, t_{2}\right)\right) \frac{(a-c)^{2}}{16}-u_{1}\left(t_{1}\right)\right]
\end{aligned}
$$

$>$ From (56) it follows that: 


$$
\begin{aligned}
& E_{\left(t_{1}, t_{2}, h_{1}, h_{2}\right)} u_{1}\left(t_{1}\right)=E_{t_{1}} u_{1}\left(t_{1}\right)=E_{t_{1}}\left[u_{1}\left(\frac{a-c}{4}\right)+\int_{\frac{a-c}{4}}^{t_{1}} u_{1}^{\prime}(x) d x\right] \\
& =E_{t_{1}}\left[u_{1}(0)+\int_{0}^{t_{1}} E_{\left(t_{2}, h_{1}, h_{2}\right)}\left[p_{1}\left(x, t_{2}\right) V_{\text {win }, 1}^{(1)}\left(x, h_{1}\left(x, t_{2}\right)\right)\right] d x\right. \\
& =u_{1}(0)+\int_{0}^{t_{1}} f\left(t_{1}\right) \int_{\frac{a-c}{4}}^{t_{1}} \int_{\frac{a-c}{4}}^{c} f\left(t_{2}\right)\left[p_{1}\left(x, t_{2}\right) E_{h_{1}} V_{w i n, 1}^{(1)}\left(x, h_{1}\left(x, t_{2}\right)\right)\right] d t_{2} d x d t_{1} \\
& =u_{1}(0)+\int_{0}^{t_{1}} \int_{x}^{c} \int_{0}^{c} f\left(t_{1}\right) f\left(t_{2}\right)\left[p_{1}\left(x, t_{2}\right) E_{h_{1}} V_{\text {win }, 1}^{(1)}\left(x, h_{1}\left(x, t_{2}\right)\right)\right] d t_{2} d t_{1} d x \\
& =u_{1}(0)+\int_{0}^{t_{1}} \int_{0}^{c} f\left(t_{2}\right)\left[p_{1}\left(x, t_{2}\right) E_{h_{1}} V_{\text {win, }, 1}^{(1)}\left(x, h_{1}\left(x, t_{2}\right)\right)\right] \int_{x}^{c} f\left(t_{1}\right) d t_{1} d t_{2} d x \\
& =u_{1}(0)+\int_{0}^{t_{1}} \int_{0}^{c} f\left(t_{2}\right)\left[p_{1}\left(x, t_{2}\right) E_{h_{1}} V_{\text {win, }, 1}^{(1)}\left(x, h_{1}\left(x, t_{2}\right)\right)\right](1-F(x)) d t_{2} d x \\
& =u_{1}(0)+\int_{0}^{t_{1}} \int_{0}^{c} f\left(t_{2}\right) f(x)\left[p_{1}\left(x, t_{2}\right) E_{h_{1}} V_{\text {win }, 1}^{(1)}\left(x, h_{1}\left(x, t_{2}\right)\right) \frac{1-F(x)}{f(x)}\right] d t_{2} d x \\
& =u_{1}(0)+\int_{0}^{t_{1}} \int_{0}^{c} f\left(t_{2}\right) f\left(t_{1}\right)\left[p_{1}\left(t_{1}, t_{2}\right) E_{h_{1}} V_{\text {win }, 1}^{(1)}\left(t_{1}, h_{1}\left(t_{1}, t_{2}\right)\right) \frac{1-F\left(t_{1}\right)}{f\left(t_{1}\right)}\right] d t_{2} d t_{1} \\
& =u_{1}(0)+E_{\left(t_{1}, t_{2}\right)}\left[p_{1}\left(t_{1}, t_{2}\right) E_{h_{1}} V_{\text {win }, 1}^{(1)}\left(t_{1}, h_{1}\left(t_{1}, t_{2}\right)\right) \frac{1-F\left(t_{1}\right)}{f\left(t_{1}\right)}\right]
\end{aligned}
$$

Substituting this last result into (109) yields:

$$
\begin{aligned}
R^{1} & =E_{\left(t_{1}, t_{2}, h_{1}, h_{2}\right)}\left[p_{1}\left(t_{1}, t_{2}\right) V_{\text {win }, 1}\left(t_{1}, h_{1}\left(t_{1}, t_{2}\right)\right)+p_{2}\left(t_{1}, t_{2}\right) V_{\text {lose }, 1}\left(t_{2}, h_{2}\left(t_{1}, t_{2}\right)\right)\right. \\
& +\left(1-p_{1}\left(t_{1}, t_{2}\right)-p_{2}\left(t_{1}, t_{2}\right)\right) \frac{(a-c)^{2}}{16} \\
& \left.-p_{1}\left(t_{1}, t_{2}\right) \frac{\left(1-F\left(t_{1}\right)\right)}{f\left(t_{1}\right)} V_{\text {win }, 1}^{(1)}\left(t_{1}, h_{1}\left(t_{1}, t_{2}\right)\right)-u_{1}(0)\right]
\end{aligned}
$$

We allow for nonzero reservation utility for the lowest type of player 1 . Of course this type will just get its reservation utility in equilibrium, we will later characterize this value. For the time being let's take these reservation utilities as given constants. We also allow for the possibility of retaining the good. In that case the overall utility of bidder 1 (gross of payment) is just $\frac{(a-c)^{2}}{16}$.

We have to take into account that if we retain the company then we still have its profit which is $\frac{(a-c)^{2}}{16}$. So we have to maximize the following expression:

$$
\begin{aligned}
& R=R^{1}+R^{2}+E_{\left(t_{1}, t_{2}\right)}\left(1-p_{1}\left(t_{1}, t_{2}\right)-p_{2}\left(t_{1}, t_{2}\right)\right) \frac{(a-c)^{2}}{16}= \\
& E_{\left(t_{1}, t_{2}, h_{1}, h_{2}\right)}\left[p _ { 1 } ( t _ { 1 } , t _ { 2 } ) \left\{V_{\text {win }, 1}\left(t_{1}, h_{1}\left(t_{1}, t_{2}\right)\right)+V_{\text {lose }, 2}\left(t_{1}, h_{1}\left(t_{1}, t_{2}\right)\right)-\frac{1-F\left(t_{1}\right)}{f\left(t_{1}\right)} V_{\text {win }, 1}^{(1)}\left(t_{1}, h_{1}\left(t_{1}, t_{2}\right)\right\}\right.\right. \\
& +p_{2}\left(t_{1}, t_{2}\right)\left\{V_{\text {win }, 2}\left(t_{2}, h_{2}\left(t_{1}, t_{2}\right)\right)+V_{\text {lose }, 1}\left(t_{2}, h_{2}\left(t_{1}, t_{2}\right)\right)-\frac{1-F\left(t_{2}\right)}{f\left(t_{2}\right)} V_{\text {win }, 2}^{(1)}\left(t_{2}, h_{2}\left(t_{1}, t_{2}\right)\right\}\right. \\
& \left.+\left(1-p_{1}\left(t_{1}, t_{2}\right)-p_{2}\left(t_{1}, t_{2}\right)\right) \frac{3(a-c)^{2}}{16}-u_{1}(0)-u_{2}(0)\right]
\end{aligned}
$$

We use here our specific functional forms and also that $\frac{1-F(t)}{f(t)}=c-t$ for the uniform distribution that is given. Then after some algebra we obtain the following expression: 


$$
\begin{gathered}
R=E_{\left(t_{1}, t_{2}, h_{1}, h_{2}\right)}\left[p _ { 1 } ( t _ { 1 } , t _ { 2 } ) \left\{\frac{2(a-c)^{2}}{9}-\frac{c(a-c)}{3}+\frac{3 t_{1}^{2}}{4}-\frac{h_{1}^{2}\left(t_{1}, t_{2}\right)}{36}+t_{1}\left(\frac{a}{2}-c\right)\right.\right. \\
\left.+\left(\frac{a-4 c}{18}\right) h_{1}\left(t_{1}, t_{2}\right)+\frac{t_{1} h_{1}\left(t_{1}, t_{2}\right)}{2}\right\}+p_{2}\left(t_{1}, t_{2}\right) \\
\begin{array}{c}
\left.\frac{2(a-c)^{2}}{9}-\frac{c(a-c)}{3}+\frac{3 t_{2}^{2}}{4}-\frac{h_{2}^{2}\left(t_{1}, t_{2}\right)}{36}+t_{2}\left(\frac{a}{2}-c\right)+\left(\frac{a-4 c}{18}\right) h_{2}\left(t_{1}, t_{2}\right)+\frac{t_{2} h_{2}\left(t_{1}, t_{2}\right)}{2}\right\} \\
\left.+\left(1-p_{1}\left(t_{1}, t_{2}\right)-p_{2}\left(t_{1}, t_{2}\right)\right) \frac{3(a-c)^{2}}{16}-u_{1}(0)-u_{2}(0)\right]
\end{array}
\end{gathered}
$$

Thus

$$
\begin{aligned}
& R=\frac{3(a-c)^{2}}{16}-u_{1}(0)-u_{2}(0)+E_{\left(t_{1}, t_{2}, h_{1}, h_{2}\right)}\left[p _ { 1 } ( t _ { 1 } , t _ { 2 } ) \left\{\frac{2(a-c)^{2}}{9}-\frac{c(a-c)}{3}-\frac{3(a-c)^{2}}{16}+\frac{3 t_{1}^{2}}{4}\right.\right. \\
& \left.-\frac{h_{1}^{2}\left(t_{1}, t_{2}\right)}{36}+t_{1}\left(\frac{a}{2}-c\right)+\left(\frac{a-4 c}{18}\right) h_{1}\left(t_{1}, t_{2}\right)+\frac{t_{1} h_{1}\left(t_{1}, t_{2}\right)}{2}\right\}+p_{2}\left(t_{1}, t_{2}\right)\left\{\frac{2(a-c)^{2}}{9}-\frac{c(a-c)}{3}-\frac{3(a-c)^{2}}{16}\right. \\
& \left.\left.+\frac{3 t_{2}^{2}}{4}-\frac{h_{2}^{2}\left(t_{1}, t_{2}\right)}{36}+t_{2}\left(\frac{a}{2}-c\right)+\left(\frac{a-4 c}{18}\right) h_{2}\left(t_{1}, t_{2}\right)+\frac{t_{2} h_{2}\left(t_{1}, t_{2}\right)}{2}\right\}\right]
\end{aligned}
$$

Now if we take the reservation utilities as given constants ${ }^{27}$ and use symmetry of our mechanism we obtain the result, after defining $D=\frac{3(a-c)^{2}}{16}-u_{1}(0)-u_{2}(0)$.

\section{Proof of Lemma 6}

Proof. Denote the (ex-ante) probability that there will be a winner (i.e. the company will be sold) by $\mathrm{P}(\mathrm{w})$, the type of the winner by $t_{w}$ and the type of the loser by $t_{l}$. Then using the symmetry of our mechanism we can rewrite the previous equation as follows (the second equality comes from the law of iterated expectations):

$$
\begin{aligned}
R & =D+P(w) E_{\left(t_{w}, t_{l}, h\right)}\left[\left\{\frac{5(a-c)^{2}}{144}-\frac{c(a-c)}{3}+\frac{3 t_{w}^{2}}{4}-\frac{h^{2}\left(t_{w}, t_{l}\right)}{36}+t_{w}\left(\frac{a}{2}-c\right)\right.\right. \\
& \left.\left.+\left(\frac{a-4 c}{18}\right) h\left(t_{w}, t_{l}\right)+\frac{t_{w} h\left(t_{w}, t_{l}\right)}{2}\right\}\right] \\
& =D+P(w) E_{t_{w}} E_{t_{l}} E_{h /\left(t_{w}, t_{l}\right)}\left[\left\{\frac{5(a-c)^{2}}{144}-\frac{c(a-c)}{3}+\frac{3 t_{w}^{2}}{4}-\frac{h^{2}\left(t_{w}, t_{l}\right)}{36}+t_{w}\left(\frac{a}{2}-c\right)\right.\right. \\
& \left.\left.+\left(\frac{a-4 c}{18}\right) h\left(t_{w}, t_{l}\right)+\frac{t_{w} h\left(t_{w}, t_{l}\right)}{2}\right\}\right] \\
& =D+P(w) E_{t_{w}}\left[\frac{5(a-c)^{2}}{144}-\frac{c(a-c)}{3}+\frac{3 t_{w}^{2}}{4}+t_{w}\left(\frac{a}{2}-c\right)\right. \\
& \left.+E_{\left(t_{l}, h\right) / t_{w}}\left\{-\frac{h^{2}\left(t_{w}, t_{l}\right)}{36}+\left(\frac{a-4 c}{18}\right) h\left(t_{w}, t_{l}\right)+\frac{t_{w} h\left(t_{w}, t_{l}\right)}{2}\right\}\right]
\end{aligned}
$$

Now by concavity in $h$ the Jensen inequality implies:

$$
\begin{aligned}
& E_{\left(t_{l}, h\right) / t_{w}}\left[-\frac{h^{2}\left(t_{w}, t_{l}\right)}{36}+\left(\frac{a-4 c}{18}\right) h\left(t_{w}, t_{l}\right)+\frac{t_{w} h\left(t_{w}, t_{l}\right)}{2}\right] \\
& \leq-\frac{\left\{E_{\left(t_{l}, h\right) / t_{w}} h\left(t_{w}, t_{l}\right)\right\}^{2}}{36}+E_{\left(t_{l}, h\right) / t_{w}}\left[\left(\frac{a-4 c}{18}\right) h\left(t_{w}, t_{l}\right)+\frac{t_{w} h\left(t_{w}, t_{l}\right)}{2}\right]
\end{aligned}
$$

\footnotetext{
${ }^{27}$ We will argue in our discussion of the main Theorem that this practice is actually valid.
} 
So,

$$
\begin{aligned}
R & \leq D+P(w) E_{t_{w}}\left[\frac{5(a-c)^{2}}{144}-\frac{c(a-c)}{3}+\frac{3 t_{w}^{2}}{4}+t_{w}\left(\frac{a}{2}-c\right)\right. \\
& \left.-\frac{\left\{E_{\left(t_{l}, h\right) / t_{w}} h\left(t_{w}, t_{l}\right)\right\}^{2}}{36}+\left(\frac{a-4 c}{18}\right) E_{\left(t_{l}, h\right) / t_{w}} h\left(t_{w}, t_{l}\right)+t_{w} E_{\left(t_{l}, h\right) / t_{w}} \frac{h\left(t_{w}, t_{l}\right)}{2}\right]
\end{aligned}
$$

Using the definition of $y\left(t_{w}\right)$ and $E_{t_{w}} y\left(t_{w}\right)(117)$ can be written in the following form:

$$
\begin{aligned}
R & \leq D+P(w) E_{t_{w}}\left[\frac{5(a-c)^{2}}{144}-\frac{c(a-c)}{3}+\frac{3 t_{w}^{2}}{4}+\frac{5 a-11 c}{9} t_{w}-\frac{y\left(t_{w}\right)^{2}}{36}+\frac{t_{w} y\left(t_{w}\right)}{2}\right] \\
& =D+2 E_{t} p(t)\left[\frac{5(a-c)^{2}}{144}-\frac{c(a-c)}{3}+\frac{3 t^{2}}{4}+\frac{5 a-11 c}{9} t-\frac{y(t)^{2}}{36}+\frac{t y(t)}{2}\right]
\end{aligned}
$$

(The last equality comes from the symmetry of our auction.)

\section{References}

\section{References}

[1] Cho, I. K. and D. M. Kreps (1987): "Signaling Games and Stable Equilibria," Quarterly Journal of Economics, 179-221

[2] Calzolari, G. and Pavan, A. (2001): "Optimal Design of Privacy Policies," http://www.spbo.unibo.it/ ${ }^{\text {calzolar/PP.pdf }}$

[3] Das Varma, G. (2000): "Bidding for a Process Innovation under Alternative Modes of Competition," http://faculty.fuqua.duke.edu/\%7edasvarma/workingpapers/index.html

[4] Fudenberg, D. and J. Tirole: "Game Theory," MIT Press

[5] Goeree, J. (2000): "Bidding for Future," mimeo, University of Virginia

[6] Jehiel, P. and B. Moldovanu (1997): "Auction with Downstream Interaction among Buyers," RAND Journal of Economics, forthcoming

[7] Jehiel, P. and B. Moldovanu (1998): "Efficient Design with Interdependent Valuations," Econometrica, forthcoming

[8] Jehiel, P. and B. Moldovanu (2000): "License Auctions and Market Structure," mimeo 
[9] Lizzeri, A (1999): "Information Revelation and Certification Intermediaries," RAND Journal of Economics, (Summer)

[10] Maskin, E. and J. Riley (1984): "Optimal Auctions with Risk Averse Buyers," Econometrica $1473-1518$

[11] Molnar, J. (2000): "Preemptive Horizontal Mergers: Theory and Evidence," mimeo, Northwestern University, http://pubweb.acns.nwu.edu/ jmo518

[12] Myerson, R. (1979): "Incentive Compatibility and the Bargaining Problem," Econometrica 61-73

[13] Myerson, R. (1981): "Optimal Auction Design," Mathematics of Operation Research, $58-73$

[14] Spence, M. (1973): "Job Market Signaling," Quarterly Journal of Economics, 355374 